\title{
ON THE DEGREE OF CONVERGENCE OF SEQUENCES OF RATIONAL FUNCTIONS*
}

\author{
BY \\ J. L. WALSH
}

The writer has recently studied $\dagger$ the convergence of certain sequences of rational functions of the complex variable, under the hypothesis that the poles of these functions are prescribed and satisfy certain asymptotic conditions. The rational functions are determined either by interpolation to a given analytic function, or by some extremal property of best approximation to such a function. Degree of convergence and regions of uniform convergence of the sequence of rational functions are then obtained (op. cit.). It is the object of the present paper to go more deeply than hitherto into properties of degree of convergence of sequences of rational functions, to make more precise the previous results, and especially to introduce and study the concept of maximal convergence of a sequence of rational functions with preassigned poles; this is a generalization of the corresponding concept for sequences of polynomials. The analogy between convergence properties of sequences of polynomials and convergence properties of sequences of more general rational functions is strong, but has hitherto not been sufficiently strong to justify the use of the term maximal convergence in the latter case (compare op. cit., p. 258). We show now that maximal convergence is characteristic of various sequences of rational functions determined by interpolation and by extremal properties. The present results would seem to be more or less definitive in form.

1. Introductory results. We choose as point of departure the following relatively simple but typical formulation (op. cit., §8.3):

Theorem 1. Let $R$ be an annular region bounded by two Jordan curves $C_{1}$ and $C_{2}$, with $C_{2}$ interior to $C_{1}$. Let the points $\alpha_{n 1}, \alpha_{n 2}, \cdots, \alpha_{n n}$ lie exterior to $C_{1}$, and let the points $\beta_{n 1}, \beta_{n 2}, \cdots, \beta_{n, n+1}$ lie on or interior to $C_{2 .}^{+}$Let $\bar{R}$ denote the

* Presented to the Society, December 30, 1938, under the title Maximal convergence of sequences of rational functions; received by the editors September 22, 1939.

$\dagger$ Interpolation and Approximation by Rational Functions in the Complex Domain, American Mathematical Society Colloquium Publications, vol. 20, New York, 1935. See especially Chapters VIII and IX. Unless otherwise indicated, all references in this paper are to this work, to which the reader should refer also for terminology.

$\ddagger$ It is a matter of taste whether or not to allow points $\alpha_{n k}$ to lie on $C_{1}$ and points $\beta_{n k}$ to lie on $C_{2}$, and whether or not to require that (1) should hold in $\bar{R}$ or on suitably restricted closed sets in $\bar{R}$. There are a variety of allowable choices here. The one we have adopted seems to the writer the most 
closure of $R$. Let the relation

$$
\lim _{n \rightarrow \infty}\left|A_{n} \frac{\left(z-\beta_{n 1}\right)\left(z-\beta_{n 2}\right) \cdots\left(z-\beta_{n, n+1}\right)}{\left(z-\alpha_{n 1}\right)\left(z-\alpha_{n 2}\right) \cdots\left(z-\alpha_{n n}\right)}\right|^{1 / n}=\Phi(z)
$$

hold uniformly on any closed set in $\bar{R}$ containing no point of $C_{2}$. Let the function $\Phi(z)$ be continuous in the closed region $\bar{R}$, and take constant values $\gamma_{1}$ and $\gamma_{2}<\gamma_{1}$ on $C_{1}$ and $C_{2}$ respectively. Denote generically* by $C_{\gamma}$ the curve $\Phi(z)=\gamma$ in $\bar{R}$, $\gamma_{2} \leqq \gamma \leqq \gamma_{1}$.

If the function $f(z)$ is analytic throughout the interior of $C_{\gamma}$ but is not analytic throughout the interior of any $C_{\gamma^{\prime}}$, with $\gamma^{\prime}>\gamma$, then we have $\left(\gamma_{2}<\lambda<\gamma\right)$

$$
\limsup _{n \rightarrow \infty}\left[\max \left|f(z)-r_{n}(z)\right|, z \text { on } C_{\lambda}\right]^{1 / n} \leqq \lambda / \gamma
$$

and we have also the limiting case of (2):

$$
\limsup _{n \rightarrow \infty}\left[\max \left|f(z)-r_{n}(z)\right|, z \text { on } C_{2}\right]^{1 / n} \leqq \gamma_{2} / \gamma,
$$

where $r_{n}(z)$ is the rational function of degree $n$ whose poles lie in the points $\alpha_{n 1}, \alpha_{n 2}, \cdots, \alpha_{n n}$, and which interpolates to $f(z)$ in the points $\beta_{n 1}, \beta_{n 2}, \cdots, \beta_{n, n+1}$.

Inequality (3) is a direct consequence of (2), by means of the relation

$$
\left[\max \left|f(z)-r_{n}(z)\right|, z \text { on } C_{2}\right] \leqq\left[\max \left|f(z)-r_{n}(z)\right|, z \text { on } C_{\lambda}\right] \text {, }
$$

and by allowing $\lambda$ in (2) to approach $\gamma_{2}$.

The form (1) obviously breaks down whenever a point $\alpha_{n k}$ is infinite, a highly important case which we do not intend to exclude. We therefore use the convention (op. cit., $\$ \S 8.1,8.2,8.5$ ) that in such an expression as the left-hand member of (1) one or more of the points $\alpha_{n k}$ may be infinite; under

convenient in view of the applications. If other choices are made, the conclusions corresponding to Theorem 1 can be read off at once from the present Theorem 1. In later parts of the present paper other choices seem more desirable. There is an obvious asymmetry in Theorem 1 relative to $\alpha_{n k}$ and $C_{1}$ on the one hand, and $\beta_{n k}$ and $C_{2}$ on the other hand. This is due to the fact that we assume $f(z)$ analytic on $C_{2}$ but not on $C_{1}$, and desire to study degree of convergence of $r_{n}(z)$ to $f(z)$ on $C_{2}$. It is then desirable to allow the points $\beta_{n k}$ to lie on (not merely within) $C_{2}$; on occasion the points $\beta_{n k}$ are to be chosen uniformly distributed on $C_{2}$. We need, however, a curve $C_{1}$ or $C_{\mu}, \gamma_{1} \geqq \mu>\gamma$, in such a relation as (4); it is desirable for explicitness to allow $\mu$ to be $\gamma_{1}$, hence desirable to assume (1) valid on $C_{1}$ and undesirable to allow points $\alpha_{n k}$ to lie on $C_{1}$. That is to say, points $\beta_{n k}$ are readily and conveniently admitted to $\bar{R}$, but not points $\alpha_{n k}$.

In Theorem 1 the demands on the location of the $\alpha_{n k}$ and $\beta_{n k}$ may without change in proof be replaced by the demands that no more than a finite number of the $\alpha_{n k}$ shall lie on or within $C_{1}$, and that the $\beta_{n k}$ shall have no limit point exterior to $C_{2}$. But if this new hypothesis is used, it may occur that for small $n$ some of the $\beta_{n k}$ lie outside of the domain of definition of $f(z)$; thus $r_{n}(z)$ need not be defined for sufficiently small $n$, but nevertheless is defined for $n$ sufficiently large.

* The notation $C_{1}$ and $C_{2}$ is exceptional to this, but no confusion should arise; there is double notation for both $C_{1}$ and $C_{2}$. 
those conditions the corresponding factors $z-\alpha_{n k}$ are simply to be omitted; and all of our formulas and conclusions remain correct without other modification.

We have hitherto (op. cit.) employed systematically condition (1) with the restriction $A_{n}=1$. Nevertheless the present more general form has appeared on occasion (for example, op. cit., pp. 206, 235, 261, 266, 274-275) and requires in the proof of Theorem 1 no change in method over the simpler form with $A_{n}=1$. Throughout the present paper we shall adopt (1) as standard.*

Even some elementary situations that are of interest are included in Theorem 1 but are not included if we require $A_{n}=1$. For instance we may take $C_{1}$ and $C_{2}$ as the circles $|z|=r_{1}$ and $|z|=r_{2}<r_{1}<r_{0}$, the numbers $\alpha_{n 1}, \cdots, \alpha_{n n}$ as the $n$th roots of an arbitrary $a_{n}$ whose modulus is not less than $r_{0}^{n}$, and the numbers $\beta_{n 1}, \cdots, \beta_{n, n+1}$ as the $(n+1)$ st roots of an arbitrary $b_{n}$ whose modulus is not greater than $r_{2}^{n+1}$. Equation (1) is valid with $A_{n}=a_{n}$, independently of the behavior of $a_{n}$ and $b_{n}$ satisfying the conditions given; but equation (1) is not valid with $A_{n}=1$ unless the numbers $\left|a_{n}\right|^{1 / n}$ approach a finite limit.

For the truth of (2) itself we assume $f(z)$ analytic throughout the interior of $C_{\gamma}$, but need not assume $f(z)$ to be analytic throughout the interior of no $C_{\gamma^{\prime}}$ with $\gamma^{\prime}>\gamma$. Indeed $f(z)$ may be analytic throughout the closed interior of $C_{\gamma_{1}}$. For our later purposes in the present paper, however, we find it desirable to make the complete assumption of Theorem 1. For appropriate examples illustrating convergence and degree of convergence when $f(z)$ is analytic throughout $R$, the reader may refer to the book already mentioned, page 239.

An interesting complement to Theorem 1 is

THEOREM 2. Under the hypothesis of Theorem 1 we have for arbitrary $\mu$, $\gamma<\mu \leqq \gamma_{1}$,

$$
\underset{n \rightarrow \infty}{\limsup }\left[\max \left|r_{n}(z)\right|, z \text { on } C_{\mu}\right]^{1 / n} \leqq \mu / \gamma .
$$

* Contrary to the situation involving asymptotic conditions for poles, there would seem to be no advantage in the study of asymptotic conditions for points $\beta_{n k}$ of interpolation in replacing the condition that

(a)

$$
\lim _{n \rightarrow \infty}\left|\left(z-\beta_{n 1}\right) \cdots\left(\beta-\beta_{n, n+1}\right)\right|^{1 / n}
$$

should exist uniformly by the condition that $\lim _{n \rightarrow \infty}\left|B_{n}\left(z-\beta_{n 1}\right) \cdots\left(z-\beta_{n, n+1}\right)\right|^{1 / n}$ should exist uniformly and not vanish identically; for it follows from Theorem 8 with $\alpha_{n k}=\infty$ that each of these conditions implies the other-this entire remark is made on the assumption that (a) is studied in the usual geometric situation, exterior to a curve within which the $\beta_{n k}$ lie. On the other hand, such a relation as (34) is ordinarily considered interior to a curve to which the $\alpha_{n k}$ are exterior. 
A formula for $r_{n}(z)$ is (op. cit., p. 186)

$$
\begin{aligned}
& r_{n}(z)=\frac{1}{2 \pi i} \int_{\Gamma}\left[1-\frac{\left(z-\beta_{n 1}\right) \cdots\left(z-\beta_{n, n+1}\right)}{\left(z-\alpha_{n 1}\right) \cdots\left(z-\alpha_{n n}\right)}\right. \\
&\left.\cdot \frac{\left(t-\alpha_{n 1}\right) \cdots\left(t-\alpha_{n n}\right)}{\left(t-\beta_{n 1}\right) \cdots\left(t-\beta_{n, n+1}\right)}\right] \frac{f(t) d t}{t-z}, \quad z \neq \alpha_{n k},
\end{aligned}
$$

where $\Gamma$ is a Jordan curve on and within which $f(z)$ is analytic, and which contains all the points $\beta_{n k}$ in its interior. Equation (5) is valid for all finite values of $z$ other than the $\alpha_{n k}$, even exterior to $\Gamma$, provided of course the integrand when not defined for such a value of $z$ is replaced by its limit for that value of $z$.

Choose the numbers $\mu_{1}, \mu_{2}$, and $\mu_{3}$ with $\mu_{1}>\mu>\gamma>\mu_{2}>\mu_{3}>\gamma_{2}$, and choose $\Gamma$ in (5) as the locus $C_{\mu_{2}}$. For $z$ on $C_{\mu}$ and $t$ on $C_{\mu_{2}}$ we have by (1) when $n$ is sufficiently large

$$
\begin{aligned}
& \left|A_{n} \frac{\left(z-\beta_{n 1}\right) \cdots\left(z-\beta_{n, n+1}\right)}{\left(z-\alpha_{n 1}\right) \cdots\left(z-\alpha_{n n}\right)}\right| \leqq \mu_{1}^{n}, \\
& \left|\frac{\left(t-\alpha_{n 1}\right) \cdots\left(t-\alpha_{n n}\right)}{A_{n}\left(t-\beta_{n 1}\right) \cdots\left(t-\beta_{n, n+1}\right)}\right| \leqq 1 / \mu_{3}^{n} .
\end{aligned}
$$

From (5) we read off at once

$$
\limsup _{n \rightarrow \infty}\left[\max \left|r_{n}(z)\right|, z \text { on } C_{\mu}\right]^{1 / n} \leqq \mu_{1} / \mu_{3},
$$

and by allowing $\mu_{1}$ to approach $\mu$ and $\mu_{3}$ to approach $\gamma$ we obtain (4).

2. Degree of convergence. We shall shortly obtain inequalities in opposite senses to (2) and (4), but in order to do this it is important to show how a certain degree of convergence on $C_{2}$ of rational functions $F_{n}(z)$ with poles in the points $\alpha_{n k}$ implies a corresponding degree of convergence on $C_{\lambda}$. The difficulty here lies in using the relation (1) directly, for the function

$$
F_{n}(z) \div A_{n} \frac{\left(z-\beta_{n 1}\right) \cdots\left(z-\beta_{n, n+1}\right)}{\left(z-\alpha_{n 1}\right) \cdots\left(z-\alpha_{n n}\right)}
$$

may have poles on $C_{2}$, and cannot be used for immediate comparison. Nevertheless we shall prove

THEOREM 3. Under the hypothesis of Theorem 1 let us suppose

$$
\limsup _{n \rightarrow \infty}\left[\max \left|F_{n}(z)\right|, z \text { on } C_{2}\right]^{1 / n}=q,
$$

* Condition (44) is a consequence of (1) and applies here directly. Nevertheless condition (1) is more elementary and more natural; it seems desirable to prove Theorem 3 without using as intermediary Theorem 12, whose proof involves a different order of ideas. 
where $F_{n}(z)$ is a rational function of degree $n$ with its poles in the points $\alpha_{n k}$. Then we have $\left(\gamma_{2}<\lambda \leqq \gamma_{1}\right)$

$$
\limsup _{n \rightarrow \infty}\left[\max \left|F_{n}(z)\right|, z \text { on } C_{\lambda}\right]^{1 / n} \leqq \lambda q / \gamma_{2} .
$$

Let $q_{1}>q$ be arbitrary, so that we have for $n$ sufficiently large $\left|F_{n}(z)\right| \leqq q_{1}^{n}$, $z$ on $C_{2}$. Let us denote by $w=\phi(z)$ a function which maps the exterior of $C_{2}$ onto $|w|>1$ so that the points at infinity correspond to each other, and denote generically by $J_{N}, N>1$, the locus $|\phi(z)|=N$ exterior to $C_{2}$. All the points $\alpha_{n k}$ lie exterior to a suitably chosen $J_{A}$, so for $Z<A, Z>1$ we have (op. cit., p. 250, Lemma I)*

$$
\left|F_{n}(z)\right| \leqq q_{1}^{n}\left[\frac{A Z-1}{A-Z}\right]^{n}
$$

By the principle of maximum for analytic functions we have for $z$ on and exterior to $J_{\boldsymbol{Z}}$

$$
\begin{aligned}
& \mid F_{n}(z) \div \\
& \quad\left\{A_{n} \frac{\left(z-\beta_{n 1}\right) \cdots\left(z-\beta_{n, n+1}\right)}{\left(z-\alpha_{n 1}\right) \cdots\left(z-\alpha_{n n}\right)}\right\} \mid \\
& \quad \leqq q_{1}^{n}\left[\frac{A Z-1}{A-Z}\right]^{n} \div\left[\min \left|A_{n} \frac{\left(z-\beta_{n 1}\right) \cdots\left(z-\beta_{n, n+1}\right)}{\left(z-\alpha_{n 1}\right) \cdots\left(z-\alpha_{n n}\right)}\right|, z \text { on } J_{Z}\right], \\
& \quad \limsup _{n \rightarrow \infty}\left[\max \left|F_{n}(z)\right|, z \text { on } C_{\lambda}\right]^{1 / n} \leqq \lambda q_{1} \frac{A Z-1}{A-Z} \div\left[\min \Phi(z), z \text { on } J_{Z}\right] .
\end{aligned}
$$

When we allow $Z$ to approach unity, the curve $J_{Z}$ approaches $C_{2}$, so by allowing $q_{1}$ to approach $q$ we obtain (7).

Our most important preliminary result is now available:

THEOREM 4. Under the hypothesis of Theorem 1 there exists no sequence of rational functions $R_{n}(z)$ of respective degrees $n$ with poles in the points $\alpha_{n k}$ such that we have either of the relations

$$
\limsup _{n \rightarrow \infty}\left[\max \left|f(z)-R_{n}(z)\right|, z \text { on } C_{2}\right]^{1 / n}=\kappa / \gamma, \quad \kappa<\gamma_{2},
$$

or $\left(\gamma_{2}<\lambda<\gamma\right)$

$$
\lim \sup \left[\max \left|f(z)-R_{n}(z)\right|, z \text { on } C_{\lambda}\right]^{1 / n}=\kappa / \gamma, \quad \kappa<\lambda .
$$

Consequently, in Theorem 1 the equality sign holds in both (2) and (3).

* In the extension ( $\$ 11)$ of Theorem 3 to a set $C_{2}$ composed of several Jordan curves, this inequality for $F_{n}(z)$ is established on a level curve $J_{Z}$ corresponding to each of the Jordan curves; the remainder of the proof holds without change. 
If we assume (8) or (9) to hold, we find by means of (3) or (2) for a specific $\lambda, \gamma_{2} \leqq \lambda<\gamma$,

$$
\limsup _{n \rightarrow \infty}\left[\max \left|r_{n}(z)-R_{n}(z)\right|, z \text { on } C_{\lambda}\right]^{1 / n} \leqq \lambda / \gamma
$$

whence from Theorem 3 for arbitrary $\mu, \gamma<\mu \leqq \gamma_{1}$,

$$
\limsup _{n \rightarrow \infty}\left[\max \left|r_{n}(z)-R_{n}(z)\right|, z \text { on } C_{\mu}\right]^{1 / n} \leqq \mu / \gamma ;
$$

a change of notation is necessary in this application of Theorem 3 if $\lambda>\gamma_{2}$. By Theorem 2 we now derive $\left(\gamma<\mu \leqq \gamma_{1}\right)$

$$
\underset{n \rightarrow \infty}{\limsup }\left[\max \left|R_{n}(z)\right|, z \text { on } C_{\mu}\right] \leqq \mu / \gamma .
$$

The function $\log \Phi(z)$ is harmonic at every point of $R$, as the uniform limit of the sequence of harmonic functions

$$
\frac{1}{n} \log \left|A_{n} \frac{\left(z-\beta_{n 1}\right) \cdots\left(z-\beta_{n, n+1}\right)}{\left(z-\alpha_{n 1}\right) \cdots\left(z-\alpha_{n n}\right)}\right|,
$$

and is continuous in $\bar{R}$, equal to $\log \gamma_{1}$ and $\log \gamma_{2}$ on $C_{1}$ and $C_{1}$ respectively.* In any closed simply connected region interior to $R$, suitably chosen conjugates of the functions (11) converge uniformly to a suitably chosen conjugate of the function $\log \Phi(z)$. As $z$ traces a curve $C_{\lambda}$ in the counterclockwise sense, the conjugate of (11), which is the argument (that is, angle or amplitude) of the function

$$
\left[\frac{\left(z-\beta_{n 1}\right) \cdots\left(z-\beta_{n, n+1}\right)}{\left(z-\alpha_{n 1}\right) \cdots\left(z-\alpha_{n n}\right)}\right]^{1 / n},
$$

increases by $2 \pi(n+1) / n$; so as $z$ traces $C_{\lambda}$ the conjugate of $\log \Phi(z)$ increases by $2 \pi$. Consequently $\Phi(z)$ is not identically constant, and we have $\gamma_{1} \neq \gamma_{2}$.

Theorem 4 is a consequence of the following theorem, a treatment of which the writer hopes to publish shortly in these Transactions. The theorem may be proved from the two-constant theorem, in a manner similar to that previously used by the present writer. $\dagger$ Theorem 5 is much more general than we need at the moment, and will be applied also later in the present paper.

THEOREM 5. Let $S$ be a region bounded by two disjoint Jordan curves $K_{0}$ and

* We cannot have $\gamma_{2}=0$, for in that case the analytic function $\exp [\log \Phi(z)+i \Psi(z)]$, where $\Psi(z)$ is conjugate to $\log \Phi(z)$ in $R$, would approach the boundary value zero everywhere on $C_{2}$, and would be locally single-valued and analytic in $R$, hence (op. cit., \$1.9) would vanish identically.

$\dagger$ Proceedings of the National Academy of Sciences, vol. 24 (1938), pp. 477-486; these Transactions, vol. 46 (1939), pp. 46-65. 
$K_{-1}$, with $K_{-1}$ interior to $K_{0}$. Let the function $u(x, y)$ be harmonic in $S$, continuous in the corresponding closed region $\bar{S}$, equal to zero and -1 on $K_{0}$ and $K_{-1}$ respectively. Denote generically by $K_{\sigma}$ the locus $u(x, y)=\sigma, 0>\sigma>-1$, and by $S_{\sigma}$ the open set $\sigma>u(x, y)>-1$ in $S$ bounded by $K_{\sigma}$ and $K_{-1}$; denote by $\bar{S}_{\sigma}$ the closure of $S_{\sigma}$.

Let the function $f(z)$ be analytic throughout $S_{\rho}$ but not analytic throughout any $S_{\rho^{\prime}}, \rho^{\prime}>\rho$, and let $f(z)$ be continuous in the two-dimensional sense on $K_{-1}$ with respect to the domain $\bar{S}$. Let the function $f_{n}(z)$ be analytic in $S$, continuous in $\bar{S}$, with the relations

$$
\begin{array}{r}
\limsup _{n \rightarrow \infty}\left[\max \left|f_{n}(z)\right|, z \text { on } K_{0}\right]^{1 / n} \leqq e^{\alpha}>1, \\
\limsup _{n \rightarrow \infty}\left[\max \left|f(z)-f_{n}(z)\right|, z \text { on } K_{-1}\right]^{1 / n} \leqq e^{\beta}<1 .
\end{array}
$$

Then we must have

$$
\alpha+\alpha \rho-\beta \rho \geqq 0
$$

if the equality sign holds here we have

$$
\begin{aligned}
& \limsup _{n \rightarrow \infty}\left[\max \left|f_{n}(z)\right|, z \text { on } K_{\mu}\right]^{1 / n}=e^{(\alpha-\beta)(\mu-\rho)}, \quad 0 \geqq \mu \geqq \rho, \\
& \text { (16) } \limsup _{n \rightarrow \infty}\left[\max \left|f(z)-f_{n}(z)\right|, z \text { on } K_{\sigma}\right]^{1 / n}=e^{(\alpha-\beta)(\sigma-\rho)}, \quad \rho>\sigma \geqq-1 \text {. }
\end{aligned}
$$

If $Q$ is an arbitrary continuum in $\bar{S}$ not a single point, and if the equality sign holds in (14), we have

$$
\underset{n \rightarrow \infty}{\limsup }\left[\max \left|f_{n+1}(z)-f_{n}(z)\right|, z \text { on } Q\right]^{1 / n}=e^{(\alpha-\beta)(U-\rho)},
$$

where $U=\max [u(x, y)$ on $Q]$. Consequently if the second member of (17) is greater than unity, the first member is equal to

$$
\limsup _{n \rightarrow \infty}\left[\max \left|f_{n}(z)\right|, z \text { on } Q\right]^{1 / n} ;
$$

if the second member of (17) is less than unity, the first member is equal to

$$
\limsup _{n \rightarrow \infty}\left[\max \left|f(z)-f_{n}(z)\right|, z \text { on } Q\right]^{1 / n} \text {. }
$$

It is a consequence of (17) and (18) that the sequence $f_{n}(z)$ converges throughout no region containing in its interior a point of $K_{\mu}, 0>\mu \geqq \rho$.

We apply Theorem 5 to the situation of Theorem 4 by identifying $C_{1}$ and $C_{\lambda}\left(\gamma_{2} \leqq \lambda<\gamma\right)$ with $K_{0}$ and $K_{-1}$ respectively. Inequalities (10) for $\mu=\gamma_{1}$ and (8) or (9) are identified with (12) and (13), so we have 


$$
\alpha=\log \gamma_{1}-\log \gamma, \quad \beta \doteq \log \kappa-\log \gamma .
$$

We also set

$$
u(x, y)=-\frac{\log \Phi(z)-\log \gamma_{1}}{\log \lambda-\log \gamma_{1}}, \quad \rho=-\frac{\log \gamma-\log \gamma_{1}}{\log \lambda-\log \gamma_{1}} .
$$

Direct substitution yields for (14) the inequality

$$
\frac{\log \gamma-\log \gamma_{1}}{\log \lambda-\log \gamma_{1}}(\log \kappa-\log \lambda) \geqq 0,
$$

whence $\kappa \geqq \lambda$, and Theorem 4 is established.

Let the equality sign hold in (14), under the conditions of Theorem 5 . If the sequence $f_{n}(z)$ converges throughout some region containing in its interior a point of $K_{\mu}, 0 \geqq \mu \geqq \rho$, it follows, from Osgood's theorem to the effect that in a region of convergence subregions of uniform convergence are everywhere dense, that the sequence $f_{n}(z)$ converges uniformly in some closed region $Q$ containing in its interior a point of some $K_{\mu^{\prime}}, 0>\mu^{\prime}>\rho$, contrary to the equality of (18) with the second member of (17).

The sequence $f_{n}(z)$ can converge like a convergent geometric series on no continuum in $\bar{S}$ exterior to $K_{\rho}$ and consisting of more than one point.

3. Maximal convergence. Theorem 4 is our chief justification for the

DeFinition. Under the hypothesis of Theorem 1, any sequence of rational functions $R_{n}(z)$ of respective degrees $n$ with poles in the points $\alpha_{n 1}, \alpha_{n 2}, \cdots, \alpha_{n n}$ is said to converge maximally to $f(z)$ on the set $C$ constituting the closed interior of $C_{2}$ provided we have

$$
\limsup _{n \rightarrow \infty}\left[\max \left|f(z)-R_{n}(z)\right|, z \text { on } C\right]^{1 / n}=\gamma_{2} / \gamma .
$$

We mention explicitly that maximal convergence is not defined if $f(z)$ is analytic throughout the interior of $C_{1}$.

As an immediate consequence of the definition we have from Theorems 1 and 4

THEOREM 6. Under the hypothesis of Theorem 1 , the sequence $r_{n}(z)$ converges maximally to $f(z)$ on $C$.

Of course the condition

$$
\limsup _{n \rightarrow \infty}\left[\max \left|f(z)-R_{n}(z)\right|, z \text { on } C_{\lambda}\right]^{1 / n} \leqq \lambda / \gamma,
$$

holding for all $\lambda$ greater than but sufficiently near $\gamma_{2}$, is sufficient for maximal convergence, for we have 


$$
\left[\max \left|f(z)-R_{n}(z)\right|, z \text { on } C_{2}\right] \leqq\left[\max \left|f(z)-R_{n}(z)\right|, z \text { on } C_{\lambda}\right],
$$

and after making the corresponding substitution we may allow $\lambda$ to approach $\gamma_{2}$.

As an alternative in the definition of maximal convergence we may replace (20) by

$$
\limsup _{z \rightarrow \infty}\left[\max \left|R_{n+1}(z)-R_{n}(z)\right|, z \text { on } C\right]^{1 / n} \leqq \gamma_{2} / \gamma,
$$

provided that the sequence $R_{n}(z)$ is assumed to converge to $f(z)$ on $C$; under this assumption we may also replace (20) by (21) with the sign $\leqq$ replaced by the equality sign. This remark is an immediate consequence of the easily proved relation

$$
\begin{aligned}
\underset{n \rightarrow \infty}{\limsup }\left[\max \left|R_{n+1}(z)-R_{n}(z)\right|, z \text { on } C\right]^{1 / n} & \\
& =\limsup _{n \rightarrow \infty}\left[\max \left|f(z)-R_{n}(z)\right|, z \text { on } C\right]^{1 / n},
\end{aligned}
$$

provided either of these expressions is less than unity.

THEOREM 7. Under the hypothesis of Theorem 1 , let $R_{n}(z)$ be a sequence of rational functions of respective degrees $n$ whose poles lie in the points $\alpha_{n k}$, and which converges maximally to $f(z)$ on $C$. Then we have $\left(\gamma_{2} \leqq \lambda<\gamma, \gamma \leqq \mu \leqq \gamma_{1}\right)$

$$
\begin{array}{r}
\limsup _{n \rightarrow \infty}\left[\max \left|f(z)-R_{n}(z)\right|, z \text { on } C_{\lambda}\right]^{1 / n}=\lambda / \gamma, \\
\quad \limsup _{n \rightarrow \infty}\left[\max \left|R_{n}(z)\right|, z \text { on } C_{\mu}\right]^{1 / n}=\mu / \gamma .
\end{array}
$$

If $Q$ is an arbitrary continuum in $\bar{R}$ not a single point, we have

(24) $\lim \sup \left[\max \left|R_{n+1}(z)-R_{n}(z)\right|, z \text { on } Q\right]^{1 / n}=[\max \Phi(z), z$ on $Q] / \gamma$.

Consequently if the second member of (24) is less than unity we have

$$
\underset{n \rightarrow \infty}{\limsup }\left[\max \left|f(z)-R_{n}(z)\right|, z \text { on } Q\right]^{1 / n}=[\max \Phi(z), z \text { on } Q] / \gamma ;
$$

if the second member of (24) is greater than or equal to unity we have

$$
\limsup _{n \rightarrow \infty}\left[\max \left|R_{n}(z)\right|, z \text { on } Q\right]^{1 / n}=[\max \Phi(z), z \text { on } Q] / \gamma .
$$

It is a consequence of (26) that the sequence $R_{n}(z)$ converges throughout no region containing in its interior a point of $C_{\mu}, \gamma_{1}>\mu \geqq \gamma$.

From the assumed maximal convergence of $R_{n}(z)$ and from the maximal convergence of $r_{n}(z)$ (Theorem 6) we have 


$$
\limsup _{n \rightarrow \infty}\left[\max \left|r_{n}(z)-R_{n}(z)\right|, z \text { on } C_{2}\right]^{1 / n} \leqq \gamma_{2} / \gamma,
$$

whence from Theorem 3

$$
\limsup _{n \rightarrow \infty}\left[\max \left|r_{n}(z)-R_{n}(z)\right|, z \text { on } C_{1}\right]^{1 / n} \leqq \gamma_{1} / \gamma .
$$

By application of Theorem 2 we may now write

$$
\limsup _{n \rightarrow \infty}\left[\max \left|R_{n}(z)\right|, z \text { on } C_{1}\right]^{1 / n} \leqq \gamma_{1} / \gamma .
$$

Inequalities (27) and (20) with $C$ replaced by $C_{2}$ place us in a position to apply Theorem 5 again. We identify $C_{1}$ and $C_{2}$ with $K_{0}$ and $K_{-1}$ respectively, and we set from (27) and (20)

$$
\alpha=\log \gamma_{1}-\log \gamma, \quad \beta=\log \gamma_{2}-\log \gamma .
$$

Moreover we have

$$
u(x, y)=-\frac{\log \Phi(z)-\log \gamma_{1}}{\log \gamma_{2}-\log \gamma_{1}}, \quad \rho=-\frac{\log \gamma-\log \gamma_{1}}{\log \gamma_{2}-\log \gamma_{1}} .
$$

Direct computation shows that the equality sign is valid in (14), and hence the conclusion of Theorem 7 follows.

Theorem 7 is a generalization and a sharpening of the corresponding result (op. cit., $\$ \$ 4.7$ and 4.8 ) for the case of approximation by polynomials; relations (24), (25), (26) are new even in the latter case. For approximation by polynomials we have $\alpha_{n k}=\infty$; according to the usual convention (op. cit., $\$ \S 8.1,8.2,8.5)$ the corresponding factors $z-\alpha_{n k}$ in (1) are simply to be omitted. With $\alpha_{n k}=\infty$ there exist (op. cit., chap. 7) various sets of points $\beta_{n k}$ for which the relation (1) obtains; for instance we may take $\Phi(z)=\Delta|\phi(z)|$, where $w=\Delta \cdot \phi(z)$ maps the exterior of $C_{2}$ onto $|w|>\Delta$ with $\phi^{\prime}(\infty)=1$; the first such set $\beta_{n k}$ was exhibited by Fejér, uniformly distributed on $C_{2}$ with respect to a suitably chosen parameter.

In connection with Theorem 7 it is worth remarking that maximal convergence of the sequence $R_{n}(z)$ to $f(z)$ on the closed interior of $C_{2}$ implies the maximal convergence of the sequence $R_{n}(z)$ to $f(z)$ on the closed interior of every $C_{\lambda}, \gamma_{2}<\lambda<\gamma$.

4. Completion of a partial sequence of $\alpha_{n k}$. Theorem 1 is valid if the $\alpha_{n k}$ and $\beta_{n k}$, and hence also the functions $r_{n}(z)$, are defined not for every $n$ but merely for an infinite sequence of indices $n$. But in the proof of Theorem 7 we have employed both $R_{n}(z)$ and $R_{n+1}(z)$, and thus have made essential use of the fact that the $R_{n}(z)$ are defined for every $n$. It is sufficient for our purposes thus far, even in the study of maximal convergence, as the reader may 
notice, if the $\alpha_{n k}$ and $R_{n}(z)$ are defined not for every $n$ but for an infinite sequence of indices $n_{j}$, with $n_{j+1}>n_{j}$ and $n_{j+1}-n_{j}$ bounded for all $j$. But if the $\alpha_{n k}$ and $R_{n}(z)$ are defined only for a sequence of indices $n_{j}$, and if $n_{j+1}-n_{j}$ is not bounded, our fundamental conclusions (22) and (23) may fail even for the specific functions $R_{n}(z) \equiv r_{n}(z)$ of Theorem 1 , as we now proceed to show by examples. These examples, chosen from Taylor's series, are closely related to gap theorems and to overconvergence in the sense of Ostrowski.

Choose $\alpha_{n k}=\infty, \beta_{n k}=0$, so that we have $\Phi(z) \equiv|z|$. Choose $C_{2}$ as the circle $|z|=1 / 2, C_{\gamma}$ as the circle $|z|=1$, and $C_{1}$ as the circle $|z|=2$. Choose the integer $n_{1}$ so that $2^{n_{1}-1}>3$, the integer $n_{2}>n_{1}$ so that $2^{\left(n_{2}-1\right) / n_{1}}>3$, and in general the integer $n_{k+1}>n_{k}$ so that $2^{\left(n_{k+1}-1\right) / n_{k}}>3$. It is sufficient to choose $n_{0}=0$, $n_{1}=3, n_{k+1}=2 n_{k}+1$. If we set

$$
f(z)=1+z^{n_{1}}+z^{n_{2}}+z^{n_{3}}+\cdots,
$$

and denote by $r_{n}(z)$ the sum of the first $n+1$ terms of the corresponding series with all of the powers of $z$ present by indication, we have by Theorem 7

$$
\limsup _{n \rightarrow \infty}\left[\max \left|f(z)-r_{n}(z)\right|, \text { for }|z|=1 / 2\right]^{1 / n}=1 / 2 \text {. }
$$

But we have for $|z|=1 / 2$

$$
\left|f(z)-r_{n_{k}}(z)\right| \leqq \frac{1}{2^{n_{k+1}}}+\frac{1}{2^{n_{k+2}}}+\cdots<\frac{1}{2^{n_{k+1}-1}}
$$

whence

$$
\limsup _{z \rightarrow \infty}\left[\max \left|f(z)-r_{n_{k}}(z)\right|, \text { for }|z|=1 / 2\right]^{1 / n_{k}} \leqq \limsup _{k \rightarrow \infty} \frac{1}{2^{\left(n_{k+1}-1\right) / n_{k}}} \leqq \frac{1}{3},
$$

in contrast to (29).

With the same choice of $\alpha_{n k}, \beta_{n k}, C_{2}, C_{1}$, and $C_{\gamma}$, let us now choose the integer $n_{1}$ so that $2^{1 /\left(n_{1}-1\right)}<3 / 2$, the integer $n_{2}>n_{1}$ so that $2^{\left(n_{1}+1\right) /\left(n_{2}-1\right)}<3 / 2$, and in general the integer $n_{k+1}$ so that $2^{\left(n_{k}+1\right) /\left(n_{k+1}-1\right)}<3 / 2$; it is sufficient to choose $n_{0}=0, n_{k+1}=2 n_{k}+3$. Again we define $f(z)$ by equation (28), and we denote by $r_{n}(z)$ the sum of the first $n+1$ terms of the corresponding series with all the powers of $z$ indicated. From Theorem 7 we have

$$
\limsup _{n \rightarrow \infty}\left[\max \left|r_{n}(z)\right|, \text { for }|z|=2\right]^{1 / n}=2 \text {. }
$$

But from (28) we may write for $|z|=2$

$$
\left|r_{n_{k}-1}(z)\right| \leqq 1+2^{n_{1}}+2^{n_{2}}+\cdots+2^{n_{k-1}} \leqq 2^{n_{k-1}+1},
$$

whence 


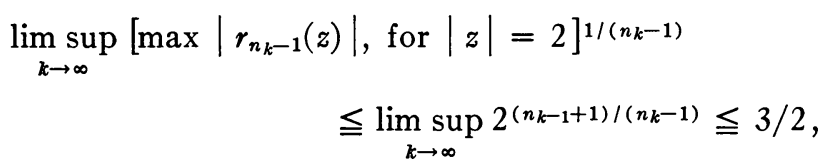

in contrast to (30).

The examples just formulated, in connection with such inequalities as (2), (3), and (4), suggest the following problem. Let $C_{1}, C_{2}, R, \Phi(z)$ be given so that the conditions of Theorem 1 are satisfied, including equation (1) but where the $\alpha_{n k}$ and $\beta_{n k}$ are defined not for all $n$, only for an infinite sequence of indices; naturally, in equation (1) only those indices are admitted. To define now new numbers $\alpha_{n k}$ and $\beta_{n k}$ where necessary so that equation (1) shall hold for all $n$. We proceed now to the discussion and solution of this problem. In the solution we need the following preliminary result:

THEOREM 8. Let the relation for some infinite sequence of indices $n$

$$
\lim _{n \rightarrow \infty}\left|A_{n} \frac{\left(z-\beta_{n 1}\right) \cdots\left(z-\beta_{n, n+1}\right)}{\left(z-\alpha_{n 1}\right) \cdots\left(z-\alpha_{n n}\right)}\right|^{1 / n}=e^{U(x, y)}
$$

be valid uniformly on every closed set interior to an annular region $R$ bounded by Jordan curves $C_{1}$ and $C_{2}$, with $C_{2}$ interior to $C_{1}$, where each $\beta_{n k}$ lies on or interior to $C_{2}$ and each $\alpha_{n k}$ lies on or exterior to $C_{1}$. Then the function $U(x, y)$ is harmonic* in $R$ and not identically constant in $R$; indeed we have

$$
\int_{\Gamma} \frac{\partial U}{\partial \nu} d s=2 \pi
$$

where $\nu$ indicates the exterior normal, where $\Gamma$ is an arbitrary analytic Jordan curve separating $C_{1}$ and $C_{2}$, and where integration is in the counterclockwise sense. At every finite point exterior to $C_{2}$ we have

$$
\begin{aligned}
& \lim _{n \rightarrow \infty}\left|\left(z-\beta_{n 1}\right) \cdots\left(z-\beta_{n, n+1}\right)\right|^{1 / n} \\
&=\exp \left[\frac{1}{2 \pi} \int_{\Gamma_{2}}\left(U \frac{\partial \log r}{\partial \nu}-\log r \frac{\partial U}{\partial \nu}\right) d s\right], \quad z=x+i y,
\end{aligned}
$$

uniformly on any closed finite set exterior to $C_{2}$, where $\Gamma_{2}$ is an analytic Jordan curve in $R$ containing $C_{2}$ but not $(x, y)$ in its interior; at every point $(x, y)$ interior to $C_{1}$ we have

* The left-hand member of (31) if existent uniformly on any closed set in $R$ vanishes at every point of $R$ or at no point of $R$. For the corresponding analytic functions are locally single-valued, different from zero, and form a normal family in any simply connected subregion of $R$. By Hurwitz's theorem any limit function of the family vanishes at every point or at no point of $R$. 


$$
\begin{aligned}
& \lim _{n \rightarrow \infty}\left|\left(z-\alpha_{n 1}\right) \cdots\left(z-\alpha_{n n}\right) / A_{n}\right|^{1 / n} \\
&=\exp \left[\frac{-1}{2 \pi} \int_{\Gamma_{1}}\left(U \frac{\partial \log r}{\partial \nu}-\log r \frac{\partial U}{\partial \nu}\right) d s\right], \quad z=x+i y,
\end{aligned}
$$

uniformly on any closed set interior to $C_{1}$, where $\Gamma_{1}$ is an analytic Jordan curve in $R$ containing $(x, y)$ and $C_{2}$ but not $C_{1}$ in its interior. In (33) and (34) the limits are to be taken over the given sequence of indices for which (31) holds. The integrals over $\Gamma_{1}$ and $\Gamma_{2}$ are to be taken in the counterclockwise and clockwise senses respectively, and $\nu$ denotes exterior and interior normal respectively.

Theorem 8 is only a slight modification of a previous result (Theorem 18, op. cit., p. 266), and the proof is therefore left to the reader. In the direction of a converse we have

TheOREM 9. Let $R$ denote an annular region bounded by Jordan curves $C_{1}$ and $C_{2}$ with $C_{2}$ interior to $C_{1}$. Let the function $U(x, y)$ be harmonic interior to $R$, continuous in the corresponding closed region, taking the values $g_{1}$ and $g_{2}<g_{1}$ on $C_{1}$ and $C_{2}$ respectively, and satisfying equation (32), where $\Gamma$ is an arbitrary analytic Jordan curve separating $C_{1}$ and $C_{2}$. Then for every $n$ there exist points $\alpha_{n k}^{\prime}$ on $C_{1}$ and points $\beta_{n \mathbf{k}}^{\prime}$ on $C_{2}$ and constants $A_{n}$ such that we have

$$
\lim _{n \rightarrow \infty}\left|A_{n} \frac{\left(z-\beta_{n 1}^{\prime}\right) \cdots\left(z-\beta_{n, n+1}^{\prime}\right)}{\left(z-\alpha_{n 1}^{\prime}\right) \cdots\left(z-\alpha_{n n}^{\prime}\right)}\right|^{1 / n}=e^{U(x, y)}
$$

uniformly on any closed set interior to $R$.

If we replace $A_{n}$ in equation (35) by $q^{n} A_{n}$, where $q$ is positive, the equation persists with $U(x, y)$ replaced by $U(x, y)+\log q$. In particular, then, it is no loss of generality to assume $g_{1}=0$ in the proof of Theorem 9; we make this assumption.

To exhibit the points $\alpha_{n k}^{\prime}$ and $\beta_{n k}^{\prime}$ desired it is now sufficient to choose the $\alpha_{n k}^{\prime}$ and $\beta_{n k}^{\prime}$ uniformly distributed on $C_{1}$ and $C_{2}$ respectively with respect to the parameter $\sigma$, where

$$
d \sigma=\frac{\partial U}{\partial \nu} d s \text { on } C_{1}, \quad d \sigma=-\frac{\partial U}{\partial \nu} d s \text { on } C_{2},
$$

for we have the equation for $(x, y)$ in $R$

$$
U(x, y)=\frac{1}{2 \pi} \int_{C_{2}} \log r d \sigma-\frac{1}{2 \pi} \int_{C_{1}} \log r d \sigma .
$$

These equations for $d \sigma$ and $U(x, y)$ still have a meaning in an extended sense even if the Jordan curves $C_{1}$ and $C_{2}$ are not analytic, and so also does the con- 
cept of uniform distribution of points on $C_{1}$ and $C_{2}$; compare op. cit., $\$ \$ 7.6$ and 9.12. Further details of the proof of Theorem 9 are so similar to a proof given elsewhere (Theorem 9, op. cit., pp. 210-211) that they are omitted here. Equation (35) is valid with $A_{n}=1$ and with $U(x, y)$ replaced by $U(x, y)-g_{1}$, hence is valid in the original form with $A_{n}=e^{n g_{1}}$.

We are now in a position to solve the proposed problem:

THEOREM 10. Under the hypothesis of Theorem 8 , where $U(x, y)$ takes constant values on $C_{1}$ and $C_{2}$, new numbers $\alpha_{n k}$ and $\beta_{n k}$ can be defined for those values of $n$ for which the original $\alpha_{n k}$ and $\beta_{n k}$ are not employed in (31), in such a way that (31) holds uniformly on any closed set interior to $R$ for the entire sequence $n=1,2,3, \cdots$.

The original sequences $\alpha_{n k}$ and $\beta_{n k}$ used in (31) yield by Theorem 8 a function $U(x, y)$ which assumes constant values $g_{1}$ and $g_{2}$ on $C_{1}$ and $C_{2}$, and which satisfies (32), where $\Gamma$ is an arbitrary analytic Jordan curve separating $C_{1}$ and $C_{2}$, and where $\nu$ indicates exterior normal. It then follows that we have $g_{2}<g_{1}$. By virtue of Theorem 9 there exist for every $n$ points $\alpha_{n k}^{\prime}$ and $\beta_{n k}^{\prime}$ which satisfy (35), uniformly on any closed set interior to $R$. For the values of $n$ that do not appear in the sequence in the relation (31) of our hypothesis we now define $\alpha_{n k}=\alpha_{n k}^{\prime}, \beta_{n k}=\beta_{n k}^{\prime}$ for those values of $n$. Then the points $\alpha_{n k}$ and $\beta_{n k}$ are now defined for every $n$, and equation (31) is valid uniformly on any closed set interior to $R$ for the complete sequence $n=1,2,3, \cdots$.

Theorem 10 applies directly in the situation of Theorem 1 but where (1) is assumed merely for a suitable sequence of indices $n$. But it is to be noticed that in Theorem 1 equation (1) is assumed to hold uniformly on $C_{1}$, whereas in Theorem 10 equation (1) holds uniformly merely on any closed set interior to $R$.

Proof of Theorem 10 by means of Theorems 8 and 9 is essentially the execution of a program previously outlined (op. cit., p. $268 \mathrm{ff}$.).

5. Determination of the $\beta_{n k}$ when $C$ and the $\alpha_{n k}$ are given. Our definition of maximal convergence involves the points $\alpha_{n k}$, the point set $C$, and the function $\Phi(z)$, but does not involve the points $\beta_{n k}$ directly; of course the $\beta_{n k}$ are intimately related to the function $\Phi(z)$. This raises the question of the determination of $\Phi(z)$ and the $\beta_{n k}$ when $C$ and the $\alpha_{n k}$ are given, a question that we proceed to discuss.

By Theorem 8, condition (34) is a consequence of (31), so (34) or some similar relation is the natural hypothesis for us to use on the points $\alpha_{n k}$. Such a condition as (34) is fulfilled whenever the points $\alpha_{n k}$ are uniformly distributed on a Jordan curve with respect to a continuous parameter.

Theorem 11. Let the point set $C$ be the closed interior of a Jordan curve $C_{2}$. 
Let $C_{1}^{\prime}$ be a Jordan curve containing in its interior the set $C$ but none of the points $\alpha_{n k}$, and let us suppose

$$
\lim _{n \rightarrow \infty}\left|\left(z-\alpha_{n 1}\right) \cdots\left(z-\alpha_{n n}\right) / A_{n}\right|^{1 / n}=e^{U(x, y)}
$$

interior to $C_{1}^{\prime}$, uniformly on any closed set interior to $C_{1}^{\prime}$. Then there exists a region $R$ bounded by $C_{2}$ and by a Jordan curve $C_{1}$ containing $C_{2}$ in its interior, and there exists a function $V(x, y)$ harmonic in $R$, continuous in the corresponding closed region, constant on $C_{1}$ and on $C_{2}$; for suitably chosen points $\beta_{n k}$ on $C_{2}$ we have interior to $R$

$$
\lim _{n \rightarrow \infty}\left|A_{n} \frac{\left(z-\beta_{n 1}\right) \cdots\left(z-\beta_{n, n+1}\right)}{\left(z-\alpha_{n 1}\right) \cdots\left(z-\alpha_{n n}\right)}\right|^{1 / n}=e^{V(x, y)},
$$

uniformly on any closed set interior to $R$.

The function $U(x, y)$ can be written as the limit of the sequence of harmonic functions

$$
U_{n}(x, y) \equiv(1 / n) \log \left|\left(z-\alpha_{n 1}\right) \cdots\left(z-\alpha_{n n}\right) / A_{n}\right|
$$

uniformly on any closed set interior to $C_{1}^{\prime}$, so $U(x, y)$ is harmonic interior to $C_{1}^{\prime}$.

Let $G(x, y)$ denote Green's function for the complement $K$ of $C$ with pole at infinity; thus $G(x, y)-\frac{1}{2} \log \left(x^{2}+y^{2}\right)$ is harmonic at infinity, and $G(x, y)$ vanishes on $C_{2}$. Let $W(x, y)$ denote the unique function which is harmonic in $K$ (even at infinity), continuous in the corresponding closed region, and equal to $U(x, y)$ on $C_{2}$. Introduce the notation

$$
V^{\prime}(x, y)=W(x, y)+G(x, y)-U(x, y),
$$

so that $V^{\prime}(x, y)$ is harmonic in the annular region $R^{\prime}$ bounded by $C_{1}^{\prime}$ and $C_{2}$, and is continuous with the value zero on $C_{2}$. If $\Gamma$ denotes an arbitrary analytic Jordan curve separating $C_{1}^{\prime}$ and $C_{2}$, and if $\nu$ denotes exterior normal, we have

$$
\int_{\Gamma} \frac{\partial V^{\prime}}{\partial \nu} d s=\int_{\Gamma} \frac{\partial W}{\partial \nu} d s+\int_{\Gamma} \frac{\partial G}{\partial \nu} d s-\int_{\Gamma} \frac{\partial U}{\partial \nu} d s=2 \pi ;
$$

for the first and third integrals of the second member vanish because $W(x, y)$ and $U(x, y)$ are harmonic respectively in the closed exterior and closed interior of $\Gamma$.

We shall now show* that $V^{\prime}(x, y)$ is positive at every point of $R^{\prime}$. Consider

* The entire proof of Theorem 11 can be interpreted as the carrying out in detail of a method previously indicated (op. cit., p. 269). But the inequality $V^{\prime}>0$ is an indispensable condition for the validity of that method, and the present proof of that inequality is the first ever given of wide generality. 
the function (compare (39))

$$
V_{n}^{\prime}(x, y)=W(x, y)+G(x, y)-U_{n}(x, y),
$$

which approaches $V^{\prime}(x, y)$ uniformly on any closed set in $R^{\prime}+C_{2}$. The function $V_{n}^{\prime}(x, y)$ is defined and harmonic at every point exterior to $C_{2}$, except the points $\alpha_{n k}$ and except at infinity. When $(x, y)$ approaches a finite point $z=\alpha_{n k}$, the functions $-U_{n}(x, y)$ and $V_{n}^{\prime}(x, y)$ become positively infinite. When $(x, y)$ becomes infinite, the functions $W(x, y), G(x, y)-\frac{1}{2} \log \left(x^{2}+y^{2}\right)$, and $\frac{1}{2} \log \left(x^{2}+y^{2}\right)-U_{n}(x, y)$ approach finite limits, except that $\frac{1}{2} \log \left(x^{2}+y^{2}\right)$ $-U_{n}(x, y)$ becomes positively infinite if one or more of the points $\alpha_{n k}$ are infinite. Consequently the minimum of the function $V_{n}^{\prime}(x, y)$ considered in the entire closed region exterior to $C_{2}$ exists and occurs for $(x, y)$ on the curve $C_{2}$ itself:

$$
\left[V_{n}^{\prime}(x, y) \text {, for }(x, y) \text { exterior to } C_{2}\right] \geqq\left[\min V_{n}^{\prime}(x, y) \text {, on } C_{2}\right] \text {. }
$$

By the uniformity of convergence of $V_{n}^{\prime}(x, y)$ to $V^{\prime}(x, y)$ on $C_{2}$ as $n$ becomes infinite, this right-hand member approaches the minimum of $V^{\prime}(x, y)$ on $C_{2}$; thus

$$
\left[V^{\prime}(x, y) \text {, for }(x, y) \text { in } R^{\prime}\right] \geqq\left[\min V^{\prime}(x, y) \text {, on } C_{2}\right] \text {. }
$$

Consequently for $(x, y)$ in $R^{\prime}$ we have proved $V^{\prime}(x, y) \geqq 0$. The function $V^{\prime}(x, y)$ is zero on $C_{2}$, can approach no negative value as $(x, y)$ in $R^{\prime}$ approaches $C_{1}^{\prime}$, and is harmonic in the region bounded by $C_{1}^{\prime}$ and $C_{2}$. But $V^{\prime}(x, y)$ is not identically zero in $R^{\prime}$, by equation (40). It follows from the well known properties of the minima of harmonic functions that the inequality $V^{\prime}(x, y)$ $>0$ persists throughout $R^{\prime}$.

We shall now exhibit the desired points $\beta_{n k}$. Let us suppose for the moment that $C_{2}$ is an analytic Jordan curve. For $(x, y)$ exterior to $C_{2}$ we have (op. cit., p. 266, Lemma IV)

$$
\begin{aligned}
& W(x, y)+G(x, y) \\
& =\frac{1}{2 \pi} \int_{C_{2}}\left[(W+G) \frac{\partial \log r}{\partial \nu}-\log r \frac{\partial(W+G)}{\partial \nu}\right] d s+q,
\end{aligned}
$$

where $\nu$ indicates the interior normal for $C_{2}$, and where $q$ is suitably chosen. For $(x, y)$ exterior to $C_{2}$ we also have (op. cit., p. 265, Lemma III)

$$
0=\frac{1}{2 \pi} \int_{C_{2}}\left(U \frac{\partial \log r}{\partial \nu}-\log r \frac{\partial U}{\partial \nu}\right) d s ;
$$

this equation is first established with $U$ replaced by $U_{n}$, and then use is made 
of the uniformity of convergence of $U_{n}$ and $\partial U_{n} / \partial \nu$ on $C_{2}$. It follows by subtraction from (41) for $(x, y)$ exterior to $C_{2}$ that

$$
\begin{aligned}
W(x, y)+G(x, y) & =\frac{1}{2 \pi} \int_{C_{2}}\left(V^{\prime} \frac{\partial \log r}{\partial \nu}-\log r \frac{\partial V^{\prime}}{\partial \nu}\right) d s+q \\
& =\frac{-1}{2 \pi} \int_{c .} \log r \frac{\partial V^{\prime}}{\partial \nu} d s+q .
\end{aligned}
$$

Thanks to the inequality $V^{\prime}(x, y)>0$ in $R^{\prime}$, we have on $C_{2}$ the inequality $d \sigma=-\left(\partial V^{\prime} / \partial \nu\right) d s \geqq 0$. If now the points $\beta_{n k}$ are chosen uniformly distributed on $C_{2}$ with respect to the parameter $\sigma$, we may write from (42) and from $-\int_{C_{2}}\left(\partial V^{\prime} / \partial \nu\right) d s=2 \pi$ (a consequence of $(40)$ ) the relation

$$
\lim _{n \rightarrow \infty}\left|\left(z-\beta_{n 1}\right) \cdots\left(z-\beta_{n, n+1}\right)\right|^{1 / n}=e^{W(x, y)+G(x, y)-q}
$$

for $z$ exterior to $C_{2}$, uniformly on any closed set exterior to $C_{2}$ (compare op. cit., §8.7). It follows now from (37) and (43) by the choice $V(x, y)=V^{\prime}(x, y)$ $-q$ that equation (38) is valid uniformly on any closed set interior to $R^{\prime}$.

In (41) and later equations we have for the sake of convenience assumed $C_{2}$ to be an analytic Jordan curve. It is sufficient if $C_{2}$ is an arbitrary Jordan curve, provided the integrals are interpreted in an extended sense (compare op. cit., \$7.6). Even if $C_{2}$ is a still more general set, the integrals may be taken over analytic Jordan curves near but exterior to $C_{2}$ on which $V(x, y)$ is constant (which does not alter the validity of (42) or of the other equations), and the points $\beta_{n k}$ may be chosen on these curves by the method of op. cit., $\$ 4.4$; but here we must relax the requirement that the $\beta_{n k}$ shall lie on or interior to $C_{2}$, and may replace it by the requirement that the $\beta_{n k}$ shall have no limit point exterior to $C_{2}$.

A region $R$ satisfying the requirements of Theorem 11 is easily defined. Let $g_{1}$ denote the least upper bound of $V^{\prime}(x, y)$ in $R^{\prime}$, and let $g$ be arbitrary, $0<g<g_{1}$. Then the locus $C_{1}: V^{\prime}(x, y)=g$ in $R^{\prime}$ is an analytic Jordan curve; the annular region bounded by $C_{1}$ and $C_{2}$ fulfills all the conditions of Theorem 11. Our fundamental results on approximation apply only to loci $V^{\prime}(x, y)=$ const., so it is no great disadvantage to cut $R$ off along such a curve.

It is not uninteresting to note that if in Theorem 11 we assume equation (37) to hold merely for an infinite sequence of indices $n$, then (as in Theorem 10) new points $\alpha_{n k}$ can be provided, and also the points $\beta_{n k}$, so that both the $\alpha_{n k}$ and $\beta_{n k}$ shall be defined for every $n$ with (38) valid. Indeed, the definition of $U(x, y)$ and the proof of (43) do not assume the $\alpha_{n k}$ defined for every $n$; Theorem 11 can be used to determine the $\beta_{n k}$ for the values of $n$ for which 
the $\alpha_{n k}$ are defined, and then Theorem 10 applies. Of course equation (43) is substantially the same as equation (33) of Theorem 8 .

6. Invariant formulation of results. The general problem of greatest degree of approximation to a given analytic function $f(z)$ by rational functions with prescribed poles $\alpha_{n k}$, culminating in Theorem 7, is invariant under linear transformation of the complex variable. But our fundamental condition (1) is not invariant, and has no obvious invariant properties. We proceed to discuss conditions analogous to (1) but having properties of invariance.

THEOREM 12. Under the conditions of Theorem 1 (it is sufficient if (1) holds uniformly merely on any closed set in $R$ ) let the function $w=\phi(z)$ map the exterior of $C_{2}$ onto $|w|>1$ so that the points at infinity correspond to each other. Then we have

$$
\lim _{n \rightarrow \infty}\left|\frac{\left[\bar{\phi}\left(\alpha_{n 1}\right) \phi(z)-1\right] \cdots\left[\bar{\phi}\left(\alpha_{n n}\right) \phi(z)-1\right]}{\left[\phi(z)-\phi\left(\alpha_{n 1}\right)\right] \cdots\left[\phi(z)-\phi\left(\alpha_{n n}\right)\right]}\right|^{1 / n}=\Phi_{1}(z),
$$

uniformly on any closed set in $R$, where $\Phi_{1}(z)$ is a suitable constant multiple of the function $\Phi(z)$ of (1).

From condition (1) it follows that we have (34) fulfilled; it follows from (34) (method of op. cit., §9.12) that

$$
\lim _{n \rightarrow \infty}\left|\left[\phi(z)-\phi\left(\alpha_{n 1}\right)\right] \cdots\left[\phi(z)-\phi\left(\alpha_{n n}\right)\right] / A_{n}\right|^{1 / n}
$$

exists uniformly on any closed set interior to $R$. By another method previously employed (op. cit., \$9.4) it follows that (44) is valid uniformly on any closed set in $R$, where $\Phi_{1}(z)$ is suitably chosen. It is to be noted that the function whose absolute value appears in (44) can be considered defined even on $C_{2}$, and to be continuous there, whence $\Phi_{1}(z)$ also is continuous on $C_{2}$ : $\Phi_{1}(z) \equiv 1$ on $C_{2}$. Then the limit in (44) can be considered uniform on any closed set in $\bar{R}$ containing no point of $C_{1}$. The function whose absolute value appears in (44) is greater than unity at every point exterior to $C_{2}$, so we have $\Phi_{1}(z) \geqq 1$ in $R$.

From (1) and (44) we may write

$$
\begin{aligned}
\lim _{n \rightarrow \infty} \mid A_{n} \frac{\left(z-\beta_{n 1}\right) \cdots\left(z-\beta_{n, n+1}\right)}{\left(z-\alpha_{n 1}\right) \cdots\left(z-\alpha_{n n}\right)} & \\
& \left.\frac{\left[\phi(z)-\phi\left(\alpha_{n 1}\right)\right] \cdots\left[\phi(z)-\phi\left(\alpha_{n n}\right)\right]}{\left[\bar{\phi}\left(\alpha_{n 1}\right) \phi(z)-1\right] \cdots\left[\bar{\phi}\left(\alpha_{n n}\right) \phi(z)-1\right]}\right|^{1 / n}=\frac{\Phi(z)}{\Phi_{1}(z)},
\end{aligned}
$$

uniformly on any closed set in $R$. Denote by $\Psi_{n}(z)\left(z-\beta_{n 1}\right)$ the function whose modulus occurs in (45). Then $\Psi_{n}(z)$ is analytic and different from zero at 
every point of the extended plane exterior to $C_{2}$, when the function is suitably defined in the points $\alpha_{n k}{ }^{*}$ Moreover $\lim _{n \rightarrow \infty}\left|z-\beta_{n 1}\right|^{1 / n}=1$ uniformly on any closed bounded set exterior to $C_{2}$. Then (45), being valid on an arbitrary $C_{\gamma}$, $\gamma_{1}>\gamma>\gamma_{2}$ when suitably interpreted, is valid at every finite point in the closed exterior of $C_{\gamma}$; the logarithm of the right-hand member of (45) can be considered defined and harmonic at every point of the extended plane exterior to $C_{2}$, is continuous in the corresponding closed region, and has the constant value $\log \gamma_{2}$ on $C_{2}$. Hence throughout $R$ we have $\Phi(z) / \Phi_{1}(z) \equiv \gamma_{2}$, and Theorem 12 is established.

Under the conditions of Theorem 12 it may occur that $C_{1}$ is a curve $|\phi(z)|=$ const., say $c$; under such circumstances the function

$$
\log \Phi(z)-\frac{\log \gamma_{1}-\log \gamma_{2}}{\log c} \log |\phi(z)|-\log \gamma_{2}
$$

is harmonic in $R$, continuous in $\bar{R}$, zero on $C_{1}$ and $C_{2}$, hence is zero in $\bar{R}$ :

$$
\log \Phi(z)=\frac{\log \gamma_{1}-\log \gamma_{2}}{\log c} \log |\phi(z)|+\log \gamma_{2} .
$$

But by (32) we have

$$
\int_{\Gamma} \frac{\partial \log \Phi(z)}{\partial \nu} d s=2 \pi,
$$

where $\Gamma$ is an analytic Jordan curve separating $C_{1}$ and $C_{2}$; also (op. cit., p. 71)

$$
\int_{\Gamma} \frac{\partial \log |\phi(z)|}{\partial \nu} d s=2 \pi,
$$

whence we deduce $\left(\log \gamma_{1}-\log \gamma_{2}\right) / \log c=1$, and for $z$ in $\bar{R}$,

$$
\Phi(z) \equiv \gamma_{2}|\phi(z)| .
$$

This equation is satisfied, it may be added, provided the points $\alpha_{n k}$ and $\beta_{n k}$ are distributed on $C_{1}$ and $C_{2}$ respectively uniformly with respect to the harmonic function conjugate to $\log |\phi(z)|$; see op. cit., $\$ 4.3$.

The converse of Theorem 12 can also be established:

THEOREM 13. Let $R$ be the region between and bounded by two Jordan curves $C_{1}$ and $C_{2}$, with $C_{2}$ interior to $C_{1}$. Let $w=\phi(z)$ map the exterior of $C_{2}$ onto the

* When a point $\alpha_{n k}$ lies at infinity, the function $\left[\phi(z)-\phi\left(\alpha_{n k}\right)\right] /\left[\bar{\phi}\left(\alpha_{n k}\right) \phi(z)-1\right]$ in (44) and (45) is to be replaced by its limit as $\alpha_{n k} \rightarrow \infty$, namely $1 / \phi(z)$. In accordance with the convention already made ( $\$ 1$ concerning factors $z-\alpha_{n k}$, there is here no exception in the behavior of $\Psi_{n}(z)$ even if points $\boldsymbol{\alpha}_{n k}$ are infinite. 
region $|w|>1$ so that the points at infinity correspond to each other. Let the points $\alpha_{n k}$ lie exterior to $C_{1}$, and suppose (44) valid uniformly on any closed set in $\bar{R}$ not containing a point of $C_{1}$. Then for suitably chosen numbers $A_{n}$ and suitably chosen points $\beta_{n k}$ on $C_{2}$, equation (1) is valid uniformly on any closed set in $R$, where $\Phi(z)$ is a constant multiple of $\Phi_{1}(z)$.

From equation (44) follows (op. cit., p. 274, Corollary 2) an equation of type (37), valid uniformly on any closed set interior to $C_{1}$. By Theorem 11 we now derive a condition of form (1), and inspection of the proof of Theorem 11 shows that equation (1) is valid uniformly on any closed set in $R$. It follows now from Theorem 12 that $\Phi(z)$ is a constant multiple of $\Phi_{1}(z)$, so Theorem 13 is established.

In Theorem 13 the Jordan curve $C_{1}$ naturally need not be a locus obtained by setting $\Phi(z)$ equal to a constant.

We remark (op. cit., p. 274) that condition (44), valid uniformly on any closed set in $R$, implies the existence of

$$
\lim _{n \rightarrow \infty}\left|\frac{\left(z-\alpha_{n 1}\right) \cdots\left(z-\alpha_{n n}\right)}{\phi\left(\alpha_{n 1}\right) \cdots \phi\left(\alpha_{n n}\right)}\right|^{1 / n}
$$

uniformly on any closed set in $R$; if a particular $\alpha_{n k}$ is infinite, the corresponding quotient $\left(z-\alpha_{n k}\right) / \phi\left(\alpha_{n k}\right)$ is to be replaced by the limit of that quotient as $\alpha_{n k}$ becomes infinite, namely $-1 / \phi^{\prime}(\infty)$. By virtue of (33), a consequence of Theorems 13 and 8, it now follows that equation (1) is valid uniformly on any closed set in $R$ for a suitably chosen $\Phi(z)$ provided we have

$$
A_{n}=\phi\left(\alpha_{n 1}\right) \cdots \phi\left(\alpha_{n n}\right) .
$$

The proof just given, that (44) implies the existence of

$$
\lim _{n \rightarrow \infty}\left|\phi\left(\alpha_{n 1}\right) \cdots \phi\left(\alpha_{n n}\right) \frac{\left(z-\beta_{n 1}\right) \cdots\left(z-\beta_{n, n+1}\right)}{\left(z-\alpha_{n 1}\right) \cdots\left(z-\alpha_{n n}\right)}\right|^{1 / n},
$$

shows the uniform existence of this limit merely on any closed set in $R$; the limit is different from zero there. But if (1) itself is satisfied under the conditions of Theorem 1, it then follows that for the original $A_{n}$

$$
\lim _{n \rightarrow \infty}\left|\frac{\phi\left(\alpha_{n 1}\right) \cdots \phi\left(\alpha_{n n}\right)}{A_{n}}\right|^{1 / n}
$$

exists and is different from zero. Consequently (1) with the substitution (46) made is valid uniformly on any closed set in $\bar{R}$ containing no point of $C_{2}$. It is not essentially more general to consider (1) in its original form than to consider (1) with (46) satisfied. 
Let us now consider the function $\Psi_{n}(z)$ defined in connection with (45), where $A_{n}$ is given by (46). We see by inspection that $\Psi_{n}(\infty)=1$, from which it follows that $\Phi(z) / \Phi_{1}(z)$ is identically unity. Thus we have proved

COROLLARY 1. Under the conditions of Theorem 1, we have

$$
\lim _{n \rightarrow \infty}\left|\phi\left(\alpha_{n 1}\right) \cdots \phi\left(\alpha_{n n}\right) \frac{\left(z-\beta_{n 1}\right) \cdots\left(z-\beta_{n, n+1}\right)}{\left(z-\alpha_{n 1}\right) \cdots\left(z-\alpha_{n n}\right)}\right|^{1 / n}=\Phi_{1}(z),
$$

uniformly on any closed set in $\bar{R}$ containing no point of $C_{2}$, where $\Phi_{1}(z)$ is given by (44).

If $z_{0}$ is an arbitrary point interior to $C_{1}$, the hypothesis of Theorem $8 \mathrm{im}$ plies (34) for $z=z_{0}$, so for the given sequence of indices

$$
\lim _{n \rightarrow \infty}\left|\left(z_{0}-\alpha_{n 1}\right) \cdots\left(z_{0}-\alpha_{n n}\right) / A_{n}\right|^{1 / n}
$$

exists. This limit is different from zero, by the form of (34) itself. It follows now from (31) that for the given sequence of indices

$$
\lim _{n \rightarrow \infty}\left|\frac{\left(z_{0}-\alpha_{n 1}\right) \cdots\left(z_{0}-\alpha_{n n}\right)\left(z-\beta_{n 1}\right) \cdots\left(z-\beta_{n, n+1}\right)}{\left(z-\alpha_{n 1}\right) \cdots\left(z-\alpha_{n n}\right)}\right|^{1 / n}
$$

exists uniformly on any closed set in $R$. Consequently whenever equation (1) is satisfied, that equation is also satisfied for some function $\Phi(z)$ with

$$
A_{n}=\left(z_{0}-\alpha_{n 1}\right) \cdots\left(z_{0}-\alpha_{n n}\right),
$$

where $z_{0}$ is an arbitrary point interior to $C_{1}$.

As a matter of record we formulate

COROLLARY 2. Under the hypothesis of Theorem 13, let $R_{n}(z)$ be a sequence of rational functions of respective degrees $n$, whose poles lie in the points $\alpha_{n k}$. Denote generically by $C_{\gamma}$ the curve $\Phi_{1}(z)=\gamma$ in $R$, and let the function $f(z)$ be analytic throughout the interior of $C_{\gamma}$ but not throughout the interior of any $C_{\gamma^{\prime}}$, $\gamma^{\prime}>\gamma$.

$A$ sufficient condition for the maximal convergence of the sequence $R_{n}(z)$ to $f(z)$ on $C$ (closed interior of $\left.C_{2}\right)$ is

$$
\limsup _{n \rightarrow \infty}\left[\max \left|f(z)-R_{n}(z)\right|, z \text { on } C_{2}\right]^{1 / n} \leqq 1 / \gamma ;
$$

if the inequality sign holds here, so also does the equality sign.

Condition (44) is invariant under linear transformation of the complex variable, and in some respects is therefore more advantageous than condition (1). It is obviously immaterial whether the loci $C_{\gamma}$ and the right-hand 
members of (22)-(26) are determined from $\Phi(z)$ or from $\Phi_{1}(z)$; in the future we shall use these functions interchangeably.

The method of proof of Theorem 12, namely the use of such an equation as (45), yields

CoROLlary 3. Under the conditions of Theorem 1, suppose also that points $\beta_{n \mathrm{k}}^{\prime}$ exist on or within the curve $C_{\gamma}, \gamma_{1}>\gamma \geqq \gamma_{2}$, so that the relation

$$
\lim _{n \rightarrow \infty}\left|A_{n}^{\prime} \frac{\left(z-\beta_{n 1}^{\prime}\right) \cdots\left(z-\beta_{n, n+1}^{\prime}\right)}{\left(z-\alpha_{n 1}\right) \cdots\left(z-\alpha_{n n}\right)}\right|^{1 / n}=\Phi_{2}(z) \not \equiv \text { const. }
$$

holds uniformly on any closed set in $\bar{R}^{\prime}$ containing no point of $C_{\gamma}$, where $R^{\prime}$ is the region between and bounded by $C_{1}$ and $C_{\gamma}$, and where $\Phi_{2}(z)$ is constant on $C_{\gamma}$. Then the quotient $\Phi_{2}(z) / \Phi(z)$ is identically constant in $R^{\prime}$.

We remark too that this corollary can be established by the use of Theorem 7 itself.

7. Maximal convergence of extremal sequences. We are now in a position expeditiously to treat extremal sequences.

THEOREM 14. Under the conditions of Theorem 1, let $C$ denote the closed interior of $C_{2}$ and let $R_{n}(z)$ be the (or a) rational function of degree $n$ with poles in the points $\alpha_{n k}$, of best approximation to $f(z)$ on $C$ in the sense of Tchebycheff, or in the sense of least pth powers $(p>0)$ over $C_{2}$ (assumed rectifiable), or in the sense of least pth powers $(p>0)$ as measured by a surface integral over $C$, or in the sense of least pth powers over $\gamma:|w|=1$ when $K$ is mapped onto the exterior of $\gamma$ so that the points at infinity correspond to each other, or in the sense of least pth powers over the circumference $|w|=1$ or over the closed region $|w| \leqq 1$ when $C$ is mapped onto $|w| \leqq 1$, in every case with a positive continuous weight function. Then the sequence $R_{n}(z)$ converges maximally to $f(z)$ on $C$.

Inequality (48) is established for the present sequence $R_{n}(z)$ precisely as in previous cases (op. cit., p. 264, Theorem 17; p. 254, Theorem 12). The details are left to the reader.

The restriction that the weight function be positive and continuous can be considerably lightened in the various cases; compare op. cit., §5.7.

8. Necessary conditions on the $\beta_{n k}$ for uniform convergence. We have hitherto presented such conditions as (1) and (47) as sufficient conditions on the $\beta_{n k}$ for maximal convergence. We now consider their necessity as conditions for uniform and maximal convergence.

THeorem 15. Let $C_{1}$ and $C_{2}$ be Jordan curves, with $C_{2}$ interior to $C_{1}$, and let $R$ denote the annular region between them. Let the points $\alpha_{n k}$ lie exterior to $C_{1}$ and 
satisfy the condition

$$
\lim _{n \rightarrow \infty}\left|\frac{\left[\overline{\phi\left(\alpha_{n 1}\right)} \phi(z)-1\right] \cdots\left[\overline{\phi\left(\alpha_{n n}\right)} \phi(z)-1\right]}{\left[\phi(z)-\phi\left(\alpha_{n 1}\right)\right] \cdots\left[\phi(z)-\phi\left(\alpha_{n n}\right)\right]}\right|^{1 / n}=\Phi(z)
$$

uniformly in $\bar{R}$; such a condition as (49) is satisfied whenever the condition (37) is fulfilled uniformly on and within $C_{1}$. Suppose the points $\beta_{n k}$ are given on or within $C_{2}$, and suppose the corresponding sequence of interpolating rational functions $r_{n}(z)$ of respective degrees $n$ converges uniformly to $f(z)$ on and within $C_{2}$ whenever $f(z)$ is analytic on and within $C_{2}$. Then we have uniformly on any closed set in $R$ the equation

$$
\lim _{n \rightarrow \infty}\left|\phi\left(\alpha_{n 1}\right) \cdots \phi\left(\alpha_{n n}\right) \frac{\left(z-\beta_{n 1}\right) \cdots\left(z-\beta_{n, n+1}\right)}{\left(z-\alpha_{n 1}\right) \cdots\left(z-\alpha_{n n}\right)}\right|^{1 / n}=\Phi(z) .
$$

Consequently the sequence $r_{n}(z)$ converges maximally to $f(z)$ on the closed interior of $C_{2}$ whenever $f(z)$ is dnalytic in that closed region but not analytic throughout $R$.

This theorem is due to Kalmár for the case $\alpha_{n k}=\infty$; the present method is due to the present writer (op. cit., §7.3), an extension of the method in the case $\alpha_{n k}=\infty$.

We introduce the notation

$$
\Psi_{n}(z)=\phi\left(\alpha_{n 1}\right) \cdots \phi\left(\alpha_{n n}\right) \frac{\left(z-\beta_{n 1}\right) \cdots\left(z-\beta_{n, n+1}\right)}{\phi(z)\left(z-\alpha_{n 1}\right) \cdots\left(z-\alpha_{n n}\right)} ;
$$

it is obvious that $\lim _{n \rightarrow \infty}|\phi(z)|^{1 / n}=1$ uniformly on any closed limited set exterior to $C_{2}$, so (50) is equivalent to

$$
\lim _{n \rightarrow \infty}\left|\Psi_{n}(z)\right|^{1 / n}=\Phi(z)
$$

uniformly on any closed set in $R$. The relation $\Phi(z) \geqq 1$ in $R$ follows at once from (49).

The sequence

$$
\left|\frac{\left(z-\alpha_{n 1}\right) \cdots\left(z-\alpha_{n n}\right)}{\phi\left(\alpha_{n 1}\right) \cdots \phi\left(\alpha_{n n}\right)}\right|^{1 / n}
$$

converges uniformly on any closed set in $R$ to a nonvanishing function (op. cit., p. 274). Hence the sequence $\log \left|\Psi_{n}(z)\right|^{1 / n}$ is uniformly bounded on any closed set in $R$, and forms a normal family of harmonic functions in $R$; these

* We are using here easily proved properties of sequences of harmonic and analytic functions, uniformly bounded and hence equicontinuous on any closed set interior to $R$.

We also use the fact that a uniformly convergent sequence of nonvanishing analytic functions converges to a nonvanishing analytic function unless the limit function vanishes identically. 
functions are obviously single-valued and harmonic in $R$. Denote by $\log |\Psi(z)|$ an arbitrary limit function of the family. We shall prove the

LEMma. Either $|\Psi(z)|$ approaches the constant unity as $z$ in $R$ approaches $C_{2}$ or for some points of $R$ the function $|\Psi(z)|$ has a value greater than unity and for other points of $R$ this function $|\Psi(z)|$ has a value less than unity.

A subsequence of the functions $\left|\Psi_{n}(z)\right|^{1 / n}$ approaches $|\Psi(z)|$ uniformly on any closed subset of $R$, so the corresponding sequence

$$
\left|\Psi_{n}(z) \frac{\left[\phi(z)-\phi\left(\alpha_{n 1}\right)\right] \cdots\left[\phi(z)-\phi\left(\alpha_{n n}\right)\right]}{\left[\overline{\phi\left(\alpha_{n 1}\right)} \phi(z)-1\right] \cdots\left[\overline{\phi\left(\alpha_{n n}\right)} \phi(z)-1\right]}\right|^{1 / n}
$$

approaches $|\Psi(z)| / \Phi(z)$ uniformly on any closed subset of $R$. The function whose modulus appears in (52) is analytic and different from zero exterior to $C_{2}$, even in the points $\alpha_{n k}$ when properly defined there, and has the modulus $1 /\left|\phi^{\prime}(\infty)\right|$ at infinity. The sequence (52) therefore converges uniformly on any closed set of the extended plane exterior to $C_{2}$, to the modulus of a function analytic exterior to $C_{2}$ and which has unit modulus at infinity. Thus $\log |\Psi(z) / \Phi(z)|$ has a meaning and is harmonic in the extended plane exterior to $C_{2}$, and has the value zero at infinity, even though $\Phi(z)$ and $\Psi(z)$ are not properly defined exterior to $C_{1}$.

Of course each of the quantities in (49) whose limit is $\Phi(z)$ is greater than unity in $R$; the function $\Phi(z)$ is not constant in $R$, so we have $\Phi(z)>1$ in $R$ (compare op. cit., p. 229); but $\Phi(z)$ is continuous and equal to unity on $C_{2}$. The lemma now follows from the well known properties of the maxima and minima of harmonic functions.

We introduce the notation $\omega_{n}(z)=\phi(z) \Psi_{n}(z)$. In the expansion of the function $1 /(t-z)$, where $t$ is exterior to $C_{2}$, by interpolating functions $r_{n}(z)$, we have (op. cit., §8.1)

$$
f(z)-r_{n}(z)=\frac{\omega_{n}(z)}{\omega_{n}(t)(t-z)} .
$$

If we introduce the notation

$$
M_{n}=\max \left[\left|\omega_{n}(z)\right|, z \text { on } C_{2}\right],
$$

our hypothesis implies

$$
\lim _{n \rightarrow \infty} M_{n} / \omega_{n}(t)=0 .
$$

If (51) is not satisfied, the normality of the family $\left|\Psi_{n}(z)\right|^{1 / n}$ implies that some limit function $|\Psi(z)|$ of this family is different from $\Phi(z)$. Then for 
some point $t$, which may be chosen in $R$, we have by the lemma for suitably chosen indices $n_{k}$

$$
\lim _{k \rightarrow \infty}\left|\Psi_{n_{k}}(t)\right|^{1 / n_{k}}=\Psi(t)<1
$$

We may also write

$$
\lim _{k \rightarrow \infty}\left|\omega_{n_{k}}(t)\right|^{1 / n_{k}}=\Psi(t)<1,
$$

and for suitably large index

$$
\left|\omega_{n_{k}}(t)\right|<1 \text {. }
$$

Choose this point $t$ as the value in (53). From the definition of $\omega_{n}(z)$ and from the properties of $\phi(z)$ on $C_{2}$ we have

$$
M_{n}=\max \left[\left|\omega_{n}(z)\right|, z \text { on } C_{2}\right]=\max \left[\left|\Psi_{n}(z)\right|, z \text { on } C_{2}\right] \text {. }
$$

From the properties at infinity of the function whose modulus appears in (52) we have

$$
\max \left[\left|\Psi_{n}(z)\right|, z \text { on } C_{2}\right] \geqq 1 /\left|\phi^{\prime}(\infty)\right|, \quad M_{n} \geqq 1 /\left|\phi^{\prime}(\infty)\right| .
$$

Inequalities (56) and (57) are in contradiction to (55), so equation (51) is established. The remainder of Theorem 15 is a consequence of Theorem 6 .

A result closely related to Theorem 15 , an extension of a result due to Fekete (op. cit., p. 163), is

Theorem 16. Let $C_{1}, C_{2}, R$, and the $\alpha_{n k}$ satisfy the conditions of Theorem 15 , including (49). Let the points $\beta_{n k}$ lie on or within $C_{2}$, and let $M_{n}$ be defined by (54). Then

$$
\lim _{n \rightarrow \infty} M_{n}^{1 / n}=1
$$

is a necessary and sufficient condition that the sequence of rational functions $r_{n}(z)$ of respective degrees $n$ with poles in the points $\alpha_{n k}$ and interpolating to $f(z)$ in the points $\beta_{n k}$, should converge uniformly to $f(z)$ on and within $C_{2}$ whenever $f(z)$ is analytic on and within $C_{2}$. Thus (58) is also a necessary and sufficient condition for the maximal convergence of the sequence $r_{n}(z)$ to $f(z)$ whenever $f(z)$ is analytic on and within $C_{2}$ but is not analytic throughout $R$.

If condition (58) is satisfied, the functions

$$
\left[\Psi_{n}(z) \frac{\left[\phi(z)-\phi\left(\alpha_{n 1}\right)\right] \cdots\left[\phi(z)-\phi\left(\alpha_{n n}\right)\right]}{\left[\overline{\phi\left(\alpha_{n 1}\right)} \phi(z)-1\right] \cdots\left[\overline{\left.\phi\left(\alpha_{n n}\right) \phi(z)-1\right]}\right]^{1 / n}}\right.
$$

are locally single-valued and analytic in the extended plane exterior to $C_{2}$ and 
form a normal family in that region in an extended sense (op. cit., p. 162). No limit function of the family can have a modulus in that region greater than unity, by (58). But each function of the family has a value at infinity of modulus $\left|\phi^{\prime}(\infty)\right|^{-1 / n}$, and each limit function has a value at infinity of modulus unity. Consequently every limit function of the family is of modulus unity throughout the extended plane exterior to $C_{2}$, and the sequence (52) approaches unity uniformly on any closed set exterior to $C_{2}$. Then by (49) equation (50) is valid uniformly on any closed set in $R$, which implies the uniform convergence of $r_{n}(z)$ to $f(z)$ whenever $f(z)$ is analytic on and within $C_{2}$, and the maximal convergence under these conditions if $f(z)$ is not analytic throughout $R$.

Conversely, let us now assume that the sequence $r_{n}(z)$ converges uniformly to $f(z)$ on and within $C_{2}$ whenever $f(z)$ is analytic on and within $C_{2}$. If (58) does not hold, we have from the reasoning used on the functions (59) for a suitable infinite sequence $n_{k}$ and for a suitable $\Delta>1$

$$
M_{n_{k}} \geqq \Delta^{n_{k}}>1 \text {. }
$$

By Theorem 15 equation (50) is satisfied. Choose $t$ in $R$ with $1<\Phi(t)<\Delta$. By (51) we have for sufficiently large $n$

$$
\left|\omega_{n}(t)\right| \leqq \Delta^{n}
$$

For the function $f(z)=1 /(t-z)$ inequalities (60) and (61) contradict our hypothesis (55). This contradiction proves (58), and implies the maximal convergence of $r_{n}(z)$ to $f(z)$ whenever $f(z)$ is analytic on and within $C_{2}$ but not analytic throughout $R$. Theorem 16 is established.

It should be mentioned that results closely related to Theorems 15 and 16 have been previously established by Shen (op. cit., p. 258); but the hypothesis on the $\alpha_{n k}$ is there geometric, not asymptotic, and the necessary and sufficient conditions derived, such as (50) and (58), are not for uniform convergence, but for an inequality corresponding to (2).

Theorems 15 and 16 are of interest chiefly in connection with maximal convergence. Nevertheless under the conditions of both those theorems (compare the remarks made concerning Theorem 1), whenever $f(z)$ is analytic throughout the interior of $\Phi(z)=T$ in $R$, we have

$$
\limsup _{n \rightarrow \infty}\left[\max \left|f(z)-r_{n}(z)\right|, z \text { on } C_{2}\right]^{1 / n} \leqq 1 / T .
$$

In connection with Theorems 15 and 16 we shall prove the

Corollary. Let $C_{1}, C_{2}, R$, and the $\alpha_{n k}$ satisfy the conditions of Theorem 15, including (49). Let the points $\beta_{n k}$ lie on $C_{2}$. Then a necessary and sufficient con- 
dition that the sequence of rational functions $r_{n}(z)$ of respective degrees $n$ with poles in the points $\alpha_{n k}$ and interpolating to $f(z)$ in the points $\beta_{n k}$ should converge uniformly to $f(z)$ on and within $C_{2}$ whenever $f(z)$ is analytic on and within $C_{2}$, is that the points $\beta_{n k}$ be uniformly distributed on $C_{2}$ with respect to the function conjugate to $\log \Phi(z)$ in $R$, continuous in $\bar{R}$.

The sufficiency of this condition (due to Fejér if $\alpha_{n k}=\infty$ ) is essentially contained in the proof of Theorem 11, in the light of the proof of Theorem 13. To prove the necessity of the condition, which is due to Kalmár if $\alpha_{n k}=\infty$, we note that (50) is a consequence of Theorem 15 and that (33) then follows; in (33) we may take $\Gamma_{2}$ as identical with $C_{2}$ if the integral is interpreted in an extended sense; by the fact that $U(x, y)$ is constant on $C_{2}$ we may write (33) in the form

$$
\lim _{n \rightarrow \infty}\left|\left(z-\beta_{n 1}\right) \cdots\left(z-\beta_{n, n+1}\right)\right|^{1 / n}=\exp \left[\frac{-1}{2 \pi} \int_{C_{2}} \log r \frac{\partial U}{\partial \nu} d s\right],
$$

uniformly on any closed bounded set exterior to $C_{2}$. The conclusion follows at once by methods previously set forth (op. cit., \$7.6).

On the topic of uniform distribution of points it is appropriate to establish

Theorem 17. Let $C_{1}, C_{2}, R$, and the $\alpha_{n k}$ satisfy the conditions of Theorem 8 , including (31), and let $U(x, y)$ be constant on $C_{1}$. Let the points $\alpha_{n k}$ lie on $C_{1}$. Then the $\alpha_{n k}$ are uniformly distributed on $C_{1}$ with respect to the function conjugate to $U(x, y)$ in $R$, continuous in $\bar{R}$.

Let $z_{0}$ be an arbitrary fixed point interior to $C_{1}$. In (34) we take the integral (in an extended sense) over $C_{1}$; by the fact that $U(x, y)$ is constant on $C_{1}$ we have

$$
\lim _{n \rightarrow \infty}\left|\frac{\left(z-\alpha_{n 1}\right) \cdots\left(z-\alpha_{n n}\right)}{\left(z_{0}-\alpha_{n 1}\right) \cdots\left(z_{0}-\alpha_{n n}\right)}\right|^{1 / n}=\exp \left[\frac{1}{2 \pi} \int_{C_{1}} \log \frac{r}{r^{0}} \cdot \frac{\partial U}{\partial \nu} d s\right],
$$

uniformly for $z$ on any closed set interior to $C_{1}$, where $\alpha$ is a variable on $C_{1}$ and $r=|z-\alpha|, r^{0}=\left|z_{0}-\alpha\right|$. If we set

$$
d u=\frac{1}{2 \pi} \frac{\partial U}{\partial \nu} d s
$$

we may then write

$$
\begin{gathered}
\lim _{n \rightarrow \infty} \frac{1}{n} \sum_{k=1}^{n} \log \frac{r_{n k}}{r_{n k}^{0}}=\int_{C_{1}} \log \frac{r}{r^{0}} \cdot d u, \\
r_{n k}=\left|z-\alpha_{n k}\right|, \quad r_{n k}^{0}=\left|z_{0}-\alpha_{n k}\right| .
\end{gathered}
$$


By virtue of (32), this integral over $C_{1}$ can be considered an integral over the interval $0 \leqq u \leqq 1$. An arbitrary function of $\alpha$ continuous on $C_{1}$ can be uniformly approximated on $C_{1}$ as closely as desired by a suitably chosen constant plus a linear combination of functions

$$
\log \left[\left|z_{k}-\alpha\right| /\left|z_{0}-\alpha\right|\right],
$$

where the $z_{k}$ are suitably chosen points interior to $C_{1}$; this follows by the transformation $z^{\prime}=1 /\left(z_{0}-\alpha\right)$ from a former lemma (op. cit., p. 169, Lemma II).* An arbitrary function $\chi(\alpha)$ constant on $C_{1}$ has the trivial property

$$
\lim _{n \rightarrow \infty} \frac{1}{n} \sum_{k=1}^{n} \chi\left(\alpha_{n k}\right)=\int_{C_{1}} \chi(\alpha) d u
$$

so it follows from the possibility of approximation that every function continuous on $C_{1}$ has the corresponding property. It follows (compare op. cit., $\$ \$ 7.5$ and 7.6) that the set $\alpha_{n k}$ is uniformly distributed on $C_{1}$ with respect to the parameter $u$.

9. Maximal convergence of sequences interpolating in the Fekete-Shen points. Certain sets of points, first introduced by Fekete for the case of polynomials, were used by him as points of interpolation to define polynomials converging favorably to a given analytic function on a given point set. The analogous points for the case of rational functions with preassigned poles $\alpha_{n k}$ were introduced and used by Shen (op. cit., §9.7) when the $\alpha_{n k}$ are subject to geometric conditions; Shen's results on degree of convergence, even for the case $\alpha_{n k}=\infty$, are more precise than those of Fekete. These same points were used subsequently by the present writer (op. cit., \$9.10) when the $\alpha_{n k}$ satisfy asymptotic conditions. We are now in a position to prove

Theorem 18. Let $C_{1}, C_{2}, R$, and the $\alpha_{n k}$ satisfy the conditions of Theorem 15, including (49). Let the $\beta_{n k}$ be the Fekete-Shen points for $C_{2}$. Then conditions (50) and (58) are fulfilled. Consequently whenever $f(z)$ is analytic on and within $C_{2}$ but not analytic throughout $R$, the sequence of rational functions $r_{n}(z)$ of respective degree $n$ with poles $\alpha_{n k}$ defined by interpolation to $f(z)$ in the points $\beta_{n k}$ converges maximally to $f(z)$ on and within $C_{2}$.

If $f(z)$ is analytic throughout the interior of the curve $C_{T}: \Phi(z)=T$ in $R$, we have (op. cit., p. 263, Corollary 2)

$$
\limsup _{n \rightarrow \infty}\left[\max \left|f(z)-r_{n}(z)\right|, z \text { on } C_{2}\right]^{1 / n} \leqq 1 / T .
$$

* It is essential to admit an additive constant, for otherwise no nonvanishing constant could itself be approximated; a uniformly convergent sequence of approximating functions converges to a uniform limit in the closed exterior of $C_{1}$, and each function $\log \left|\left(z_{k}-\alpha\right) /\left(z_{0}-\alpha\right)\right|$ is harmonic and vanishes at infinity: $\alpha=\infty$. 
The maximal convergence of the sequence $r_{n}(z)$ follows from Corollary 2 to Theorem 13, if $f(z)$ is not analytic throughout the interior of any $C_{T^{\prime}}, T^{\prime}>T$. The remainder of Theorem 18 follows from Theorems 15 and 16.

It is of interest to note that the Fekete-Shen points $\beta_{n k}$ lie on $C_{2}$ itself, and hence by the Corollary to Theorem 16 these points are uniformly distributed on $C_{2}$ with respect to the function conjugate to $\log \Phi(z)$ in $R$, continuous in $\bar{R}$.

It is of some interest to note that the entire theory of maximal convergence of sequences of rational functions may be developed by taking not (1) but condition (49) as fundamental, using the Fekete-Shen points (as in op. cit., \$9.10) to obtain a sequence of rational functions $r_{n}(z)$ satisfying the above inequality; the analogue of (4) is readily proved, so that Theorem 5 applies. The remainder of the theory can then be built up. It seems simpler and more natural to the writer to make (1) fundamental rather than (49).

10. A general extremal problem on approximation by functions with preassigned poles. The problem of approximation by rational functions with preassigned poles can be considered a special case of approximation by more general functions with preassigned poles.

Theorem 19. Let $C_{1}, C_{2}, R$, and the points $\alpha_{n k}$ and $\beta_{n k}$ satisfy the conditions of Theorem 1, including condition (1). Let $R_{\gamma}$ denote generically the annular region bounded by $C_{2}$ and $C_{\gamma}$. Suppose the function $F(z)$ to be analytic in $R_{\gamma}$, $\gamma_{1}>\gamma>\gamma_{2}$, but not analytic throughout any $R_{\gamma^{\prime}}, \gamma^{\prime}>\gamma ;$ suppose $F(z)$ continuous in the two-dimensional sense on $C_{2}$.

Denote by $K$ the region exterior to $C_{2}$. Then there exist functions $F_{n}(z)$ analytic in $K$ except for possible poles in the points $\alpha_{n k}$, continuous in the two-dimensional sense on $C_{2}$, such that we have

$$
\underset{n \rightarrow \infty}{\limsup }\left[\max \left|F(z)-F_{n}(z)\right|, z \text { on } C_{2}\right]^{1 / n}=\gamma_{2} / \gamma ;
$$

but there exists no such sequence $F_{n}(z)$ for which the left-hand member of (62) is less than $\gamma_{2} / \gamma$.

Any sequence $F_{n}(z)$ for which (62) holds possesses also the following properties:

$$
\begin{aligned}
& \limsup _{n \rightarrow \infty}\left[\max \left|F(z)-F_{n}(z)\right|, z \text { on } C_{\lambda}\right]^{1 / n}=\lambda / \gamma, \quad \gamma_{2} \leqq \lambda<\gamma, \\
& \limsup _{n \rightarrow \infty}\left[\max \left|F_{n}(z)\right|, z \text { on } C_{\mu}\right]^{1 / n}=\mu / \gamma, \quad \gamma \leqq \mu \leqq \gamma_{1} .
\end{aligned}
$$

If $Q$ is an arbitrary continuum in $\bar{R}$ not a single point, we have

(65) $\limsup _{n \rightarrow \infty}\left[\max \left|F_{n+1}(z)-F_{n}(z)\right|, z \text { on } Q\right]^{1 / n}=[\max \Phi(z), z$ on $Q] / \gamma$. 
The function $F(z)$ can be written as the sum of its two components:

$$
\begin{aligned}
& F(z) \equiv f_{1}(z)+f_{2}(z), \\
& f_{1}(z) \equiv \frac{1}{2 \pi i} \int_{\Gamma_{1}} \frac{F(t) d t}{t-z}, \\
& f_{2}(z) \equiv \frac{1}{2 \pi i} \int_{\Gamma_{2}} \frac{F(t) d t}{t-z},
\end{aligned}
$$$$
z \text { in } R_{\gamma} \text {, }
$$

where $\Gamma_{1}$ is a rectifiable Jordan curve in $R_{\gamma}$ containing $z$ and $C_{2}$ in its interior, and where $\Gamma_{2}$ is a rectifiable Jordan curve in $R_{\gamma}$ containing $C_{2}$ in its interior but having $z$ exterior to it; the curves $\Gamma_{1}$ and $\Gamma_{2}$ depend then on $z$, but the functions $f_{1}(z)$ and $f_{2}(z)$ are independent of the particular curves chosen. Equation $\left(66_{1}\right)$ is valid for $z$ in $R_{\gamma}$. Nevertheless the integrals in $\left(66_{2}\right)$ and $\left(66_{3}\right)$ define $f_{1}(z)$ and $f_{2}(z)$ as functions analytic respectively throughout the interior of $C_{\gamma}$ and throughout the exterior of $C_{2}$ (even at infinity, by a limiting process). Equation $\left(66_{1}\right)$ can be used to define $f_{2}(z)$ on $C_{2}$; with this additional definition, the function $f_{2}(z)$ is continuous on $C_{2}$. The function $f_{1}(z)$ is analytic throughout the interior of no $C_{\gamma^{\prime}}, \gamma^{\prime}>\gamma$.

We are now in a position to identify the function $f(z)$ of Theorem 1 with the present $f_{1}(z)$. If we write

$$
f(z) \equiv f_{1}(z), \quad r_{n}(z) \equiv F_{n}(z)-f_{2}(z),
$$

equation (62) is a consequence of (indeed identical with) equation (3) (see Theorem 4).

The method of proof of Theorems 3 and 4 applies also in the present case, and shows that there exists no sequence $F_{n}(z)$ for which the left-hand member of (62) is less than $\gamma_{2} / \gamma$.

Let us suppose now $F_{n}(z)$ to be an arbitrary sequence satisfying (62). With the aid of the comparison sequence $r_{n}(z)+f_{2}(z)$ just considered, which satisfies (62) in place of $F_{n}(z)$, we find as in the proof of (10)

$$
\limsup _{n \rightarrow \infty}\left[\max \left|F_{n}(z)\right|, z \text { on } C_{\mu}\right]^{1 / n} \leqq \mu / \gamma, \quad \gamma<\mu \leqq \gamma_{1} .
$$

Theorem 5 now applies, and yields the remaining parts of Theorem 19.

A slight modification of Theorem 19 is of interest. Let us replace the requirement that $F(z)$ be continuous in the two-dimensional sense on $C_{2}$ by the requirement that $F(z)$ be bounded in the neighborhood of $C_{2}$, with the identical modification in the requirements on $F_{n}(z)$. The expression

$$
\max \left|F(z)-F_{n}(z)\right|, \quad z \text { on } C_{2},
$$

which occurs in (62) is then to be interpreted as 


$$
\limsup _{\lambda \rightarrow \gamma_{2}}\left[\max \left|F(z)-F_{n}(z)\right|, z \text { on } C_{\lambda}\right] \text {, }
$$

which is necessarily finite. With this understanding, also similarly for (63), (64), and (65), Theorem 5 is valid for the corresponding sequences, and Theorem 19 is valid in the generalized form.

With this new convention, there exists for each $n$ at least one admissible function $F_{n}(z)$ for which $(67)$ is least. By an admissible function $F_{n}(z)$ we understand here a function analytic in $K$ except for possible poles in the points $\alpha_{n k}$, and bounded in the neighborhood of $C_{2}$. Let $N_{0}$ denote the greatest lower bound of all numbers (67) for the class of admissible functions. Let $F_{n}^{(1)}(z), F_{n}^{(2)}(z), \cdots$ be admissible functions for which the corresponding numbers $N^{(1)}, N^{(2)}, \cdots$ approach $N_{0}$ :

$$
\lim _{k \rightarrow \infty} N^{(k)}=N_{0} .
$$

We have

$$
\begin{aligned}
{[\max } & \left.\left|F_{n}^{(1)}(z)-F_{n}^{(k)}(z)\right|, z \text { on } C_{\lambda}\right] \\
\leqq & {\left[\max \left|F(z)-F_{n}^{(1)}(z)\right|, z \text { on } C_{\lambda}\right]+\left[\max \left|F(z)-F_{n}^{(k)}(z)\right|, z \text { on } C_{\lambda}\right], } \\
& \quad \limsup _{\lambda \rightarrow \gamma_{2}}\left[\max \left|F_{n}^{(1)}(z)-F_{n}^{(k)}(z)\right|, z \text { on } C_{\lambda}\right] \leqq N^{(1)}+N^{(k)} .
\end{aligned}
$$

The function $F_{n}^{(1)}(z)-F_{n}^{(k)}(z)$ is analytic in $K$ except for poles in the points $\alpha_{n k}$. The function

$$
\frac{\left[F_{n}^{(1)}(z)-F_{n}^{(k)}(z)\right]\left[\phi(z)-\phi\left(\alpha_{n 1}\right)\right] \cdots\left[\phi(z)-\phi\left(\alpha_{n n}\right)\right]}{\left[\overline{\phi\left(\alpha_{n 1}\right)} \phi(z)-1\right] \cdots\left[\overline{\phi\left(\alpha_{n n}\right)} \phi(z)-1\right]},
$$

when suitably defined in the points $\alpha_{n k}$, is analytic throughout $K$ and by (69) is uniformly bounded (independently of $k$ ) in $K$. From any infinite sequence of functions (70) can be extracted a subsequence which converges throughout $K$, uniformly on any closed set in $K$, to a bounded function of form (70) with $F_{n}^{(k)}(z)$ replaced by an admissible function $F_{n}^{(0)}(z)$. The corresponding subsequence of the $F_{n}^{(k)}(z)$ then converges to $F_{n}^{(0)}(z)$ throughout $K$ except in the points $\alpha_{n k}$, uniformly on any closed set in $K$ containing no point $\alpha_{n k}$. Let us suppose (this supposition involves merely a change of notation) that the original sequence $F_{n}^{(k)}(z)$ so converges.

Let $\mu$ be chosen arbitrarily but fixed, $\gamma>\mu>\gamma_{2}$, and let us suppose $\gamma_{2}<\lambda<\mu$, where $\lambda$ will be allowed to approach $\gamma_{2}$. We now apply the twoconstant theorem (or Hadamard three-circle theorem), which is valid under 
the present generalized conditions on the functions involved:

$$
\begin{aligned}
{[\max \mid F(z)} & \left.-F_{n}^{(k)}(z) \mid, z \text { on } C_{\lambda}\right] \\
\leqq & {\left[\max \left|F(z)-F_{n}^{(k)}(z)\right|, z \text { on } C_{2}\right]^{(\mu-\lambda) /\left(\mu-\gamma_{2}\right)} } \\
& \quad\left[\max \left|F(z)-F_{n}^{(k)}(z)\right|, z \text { on } C_{\mu}\right]^{\left(\lambda-\gamma_{2}\right) /\left(\mu-\gamma_{2}\right)} .
\end{aligned}
$$

The second square bracket in the second member of (71) has a bound $N$ independent of $k$; the first square bracket in the second member of (71) approaches $N_{0}$, by (68). Thus we have by letting $k$ become infinite

$$
\begin{aligned}
{\left[\max \left|F(z)-F_{n}^{(0)}(z)\right|, z \text { on } C_{\lambda}\right] } & =\lim _{k \rightarrow \infty}\left[\max \left|F(z)-F_{n}^{(k)}(z)\right|, z \text { on } C_{\lambda}\right] \\
& \leqq N_{0}^{(\mu-\lambda) /\left(\mu-\gamma_{2}\right)} \cdot N^{\left(\lambda-\gamma_{2}\right) /\left(\mu-\gamma_{2}\right)}
\end{aligned}
$$

the first equality sign is a consequence of the uniform convergence of $F_{n}^{(k)}(z)$ to $F_{n}^{(0)}(z)$ on $C_{\lambda}$. If we allow $\lambda$ to approach $\gamma_{2}$ in (72), we have

$$
\left[\max \left|F(z)-F_{n}^{(0)}(z)\right|, z \text { on } C_{2}\right] \leqq N_{0} ;
$$

the inequality sign is impossible here by the definition of $N_{0}$, so we have established the existence of at least one extremal function $F_{n}(z)$. The question of the uniqueness of this extremal function is still open.

Naturally the extremal functions $F_{n}(z)$ whose existence has just been established satisfy condition (62); and for them the conclusion of Theorem 19 is valid.

We remark too that the requirements of continuity of $F(z)$ and $F_{n}(z)$ on $C_{2}$ need not be replaced by the requirement of boundedness in the neighborhood of $C_{2}$ but may simply be omitted, without essentially altering our conclusions; the square bracket in the left-hand member of (62) is merely understood as (67); a suitable comparison sequence $F_{n}(z)$ can be obtained as before from Theorem 1 ; at least one extreme function $F_{n}(z)$ exists.

Whether or not this last generalization is allowed, we shall prove

TheOREm 20. Let the conditions of Theorem 19 be fulfilled, including equation (62), and let the respective components of the function $F_{n}(z)$ be $f_{n 1}(z)$ and $f_{n 2}(z)$, defined by equations analogous to (66). Then the function $f_{n 1}(z)$ is a rational function of degree $n$ with poles in the points $\alpha_{n k}$, and the sequence $f_{n 1}(z)$ converges maximally to $f_{1}(z)$ on the closed interior of $C_{2}$.

In the region $R_{\gamma}$ we have the equation

$$
F_{n}(z)=f_{n 1}(z)+f_{n 2}(z),
$$

where $f_{n 1}(z)$ is analytic throughout the interior of $C_{\gamma}$, and $f_{n 2}(z)$ is analytic 
throughout the exterior of $C_{2}$, even at infinity. But $F_{n}(z)$ is analytic throughout $K$ except in the points $\alpha_{n k}$, so $f_{n 1}(z)$ can be extended analytically from $R_{\gamma}$, and can be considered defined and analytic throughout $K$ except for poles in the points $\alpha_{n k}$. Consequently $f_{n 1}(z)$ is a rational function of degree $n$ whose poles lie in the points $\alpha_{n k}$.

In the defining equations for $f_{1}(z)$ and $f_{n 1}(z)$ we may choose $\Gamma_{1}$ as the curve $C_{\lambda}, \gamma_{2}<\lambda<\gamma$, whence

$$
f_{1}(z)-f_{n 1}(z)=\frac{1}{2 \pi i} \int_{C_{\lambda}} \frac{F(t)-F_{n}(t)}{t-z} d t, \quad z \text { on } C_{2} .
$$

Then equation (63) yields

$$
\limsup _{n \rightarrow \infty}\left[\max \left|f_{1}(z)-f_{n 1}(z)\right|, z \text { on } C_{2}\right]^{1 / n} \leqq \lambda / \gamma .
$$

In this inequality we may allow $\lambda$ to approach $\gamma_{2}$ :

$$
\limsup _{n \rightarrow \infty}\left[\max \left|f_{1}(z)-f_{n 1}(z)\right|, z \text { on } C_{2}\right]^{1 / n} \leqq \gamma_{2} / \gamma
$$

The function $f_{2}(z)$ is analytic throughout the region $K$, and $F(z)$ is analytic throughout the interior of $C_{\gamma}$ but not throughout the interior of any $C_{\gamma^{\prime}}$, $\gamma^{\prime}>\gamma$. Then $f_{1}(z)$ also is analytic throughout the interior of $C_{\gamma}$ but not throughout the interior of any $C_{\gamma^{\prime}}$. Inequality (73) thus establishes the conclusion of Theorem 20.

Results analogous to but less explicit than Theorems 19 and 20 lie at hand if we replace the original requirement on $F(z)$ by the requirement that $F(z)$ be analytic throughout $R$. For instance there exist functions $F_{n}(z)$ analytic in $K$ except for possible poles in the points $\alpha_{n k}$ such that the first member of (62) is not greater than $\gamma_{2} / \gamma_{1}$.

We have several comments to make regarding the general significance of the problem studied in Theorems 19 and 20.

$1^{\circ}$. Theorem 19 is a result concerning a problem which is invariant under arbitrary one-to-one conformal transformation of the region $K$, even under some circumstances (compare $\$ 11$ below) if $K$ is transformed into a region not bounded by a Jordan curve. But the full import of this invariant property appears best if the hypothesis (1) is replaced by the equivalent hypothesis (49).

$2^{\circ}$. The two groups, Theorems $1-4$ on the one hand and Theorems 19 and 20 on the other, are intimately related to each other, especially in view of Theorem 5. Theorem 1 can be employed as above to furnish a sequence $F_{n}(z)$ which converges as rapidly (in the sense indicated by (62)) as does any 
sequence of admissible functions. Reciprocally, Theorem 20 shows that Theorem 19 can be employed to furnish a sequence of rational functions which converges maximally.

$3^{\circ}$. Theorem 1 can be formulated so as to be invariant under arbitrary linear transformation of the complex variable. Condition (49) has the more general property of being essentially invariant under arbitrary one-to-one conformal transformation of the closed exterior of $C_{2}$. Remarks $1^{\circ}$ and $2^{\circ}$ serve to explain this latter invariant property, which has been in the past somewhat mystifying.

$4^{\circ}$. Theorem 19 is a companion piece (Gegenstück) to results previously established by the present writer concerning interpolation by functions analytic and bounded in a given simply connected region $K{ }^{*}$ Prescribed poles on the one hand correspond to prescribed points of interpolation on the other, in each case satisfying asymptotic conditions in an annular region $R$ interior to $K$, one of whose boundaries is the boundary of $K$. In the problem of interpolation there are two equivalent conditions on the prescribed points, completely analogous to (1) and (49). The function approximated to in the one problem and the function interpolated to in the other are assumed analytic in respective regions mutually complementary with respect to $K$, and convergence of approximating and interpolating functions is established throughout those (suitably chosen) regions of analyticity bounded by loci $\Phi=$ const. in $R$. Indeed, this entire pair of configurations exhibits a complete duality, in the sense already defined (op. cit., §8.3), except that the present duality is concerned with functions meromorphic or analytic in a region rather than with rational functions as in the former duality. Theorem 5 is sufficiently powerful to apply to both configurations.

11. Generalizations and extensions. In connection with the foregoing results, there suggests itself the question of extensions to regions of higher connectivity, and to regions not bounded by Jordan curves. We now proceed to discuss these questions in order.

We have hitherto limited ourselves to a region bounded merely by two Jordan curves $C_{1}$ and $C_{2}$ instead of two such sets of curves, entirely for the sake of simplicity and ease of exposition. Our methods are applicable with only minor modifications in the more general case. For instance we sketch rapidly the proof of

Theorem 21. Let $R$ be a finite region bounded by two disjoint sets $C_{1}$ and $C_{2}$, each composed of a finite number of disjoint Jordan curves; let one of the Jordan curves $C_{1}^{0}$ of the former set contain in its interior all points of $C_{1}-C_{1}^{0}+C_{2}$. Let

* These Transactions, vol. 46 (1939), pp. 46-65. 
the points $\alpha_{n 1}, \alpha_{n 1}, \cdots, \alpha_{n n}$ be separated from $R$ by $C_{1}$, and let the points $\beta_{n 1}, \beta_{n 2}, \cdots, \beta_{n, n+1}$ not lying on $C_{2}$ be separated from $R$ by $C_{2}$. Let $\bar{R}$ denote the closure of $R$.

Let the relation (1) hold uniformly on any closed set in $\bar{R}$ containing no point of $C_{2}$. Let the function $\Phi(z)$ be continuous in the closed region $\bar{R}$, and take constant values $\gamma_{1}$ and $\gamma_{2}<\gamma_{1}$ on $C_{1}$ and $C_{2}$ respectively. Denote generically by $C_{\gamma}$ the locus $\Phi(z)=\gamma$ in $\bar{R}, \gamma_{2} \leqq \gamma \leqq \gamma_{1}$. For $\gamma_{2}<\gamma<\gamma_{1}$, the locus $C_{\gamma}$ consists of a finite number of mutually disjoint analytic Jordan curves, except that for each of a finite number of values of $\gamma$ there are a finite number of multiple points, each common to a finite number of the Jordan curves composing $C_{\gamma}$. Denote generically by $R_{\gamma}$ the open set composed of the interiors of the Jordan curves composing $C_{2}$ plus the point set in $\bar{R}$ on which we have $\gamma_{2} \leqq \Phi(z)<\gamma$; thus $R_{\gamma}$ need not be connected, but is the sum of a finite number of regions bounded by the entire locus $C_{\gamma}$.

Let the function $f(z)$ be single-valued and analytic on $R_{\gamma}$ but not single-valued and analytic on any $R_{\gamma^{\prime}}$ with $\gamma^{\prime}>\gamma$. Then we have (2), (3), and (4) fulfilled, where $r_{n}(z)$ is the rational function of degree $n$ whose poles lie in the points $\alpha_{n k}$ and which interpolates to $f(z)$ in the points $\beta_{n k}$.

There exists no sequence of rational functions $R_{n}(z)$ of respective degrees $n$ with poles in the points $\alpha_{n k}$ such that either (8) or (9) is satisfied.

Whenever a set of rational functions $R_{n}(z)$ of respective degrees $n$ with poles in the points $\alpha_{n k}$ satisfies (20), we shall say that the sequence $R_{n}(z)$ converges maximally to $f(z)$ on the set $C$ composed of the Jordan curves $C_{2}$ and their respective interiors. Consequences of maximal convergence are equations (22) and (23), and if $Q$ is an arbitrary continuum in $\bar{R}$ not a single point, also equations (24)(26).

In Theorem 21 the function $f(z)$ need not be a monogenic analytic function; nevertheless $f(z)$ can be extended analytically from the neighborhood of $C_{2}$ by paths in $R_{\gamma}$ so as to be single-valued and analytic at every point of $R_{\gamma}$; but this property is shared by no set $R_{\gamma^{\prime}}$ with $\gamma^{\prime}>\gamma$. Then (a) for some path of analytic extension in $R_{\gamma}$ from $C_{2}$ to $C_{\gamma}$ the function $f(z)$ has a singularity on $C_{\gamma}$, or (b) the locus $C_{\gamma}$ has a multiple point $A$ and the analytic extensions of $f(z)$ from $C_{2}$ along paths in the various regions into which $C_{\gamma}$ falls of which $A$ is a boundary point fail to be analytic and identical throughout a neighborhood of $A$; or both (a) and (b) occur.

Theorem 5 extends to a region $R$ bounded by two sets of Jordan curves, and the original proofs already given yield now Theorem 21 .

With only minor modifications, which we now mention, the entire discussion of $\$ \$ 1-10$ of the present paper is valid for the more general region $R$ introduced in Theorem 21. 
Under the hypothesis of Theorem 12 (that is to say, the hypothesis of Theorem 1), equation (44) may be written

$$
\lim _{n \rightarrow \infty} \frac{1}{n} \sum_{k=1}^{n} G\left(z, \alpha_{n k}\right)=\log \Phi_{1}(z)
$$

uniformly on any closed set in $R$, where

$$
G\left(z, \alpha_{n k}\right)=\log \left|\frac{\bar{\phi}\left(\alpha_{n k}\right) \phi(z)-1}{\phi(z)-\phi\left(\alpha_{n k}\right)}\right|
$$

is Green's function* with pole in the point $\alpha_{n k}$ for the region exterior to the Jordan curves $C_{2}$. With this change in the manner of writing (44), the new formulation of Theorem 12 is obviously valid; theorem and proof extend without great difficulty to the situation of Theorem 21. But equation (44) is obviously simpler than (74), and more easily applied. Of course $C_{1}$ may consist of several Jordan curves even when $C_{2}$ is a single Jordan curve; under such circumstances (44) and similar relations need no revision.

Let us be more explicit about the extension of Theorem 12, using now the hypothesis of Theorem 21 rather than of Theorem 1 ; it is sufficient if (1) holds uniformly merely on any closed set in $R$. On an arbitrary closed set $C^{\prime}$ in $R$ the functions $G\left(z, \alpha_{n k}\right)$ are uniformly bounded, for the points $\alpha_{n k}$ are bounded from $C^{\prime}$, and the functions $G\left(z, \alpha_{n k}\right)$ in $R$ depend continuously on $z$ and $\alpha_{n k}$ except in the neighborhood of $\alpha_{n k}$. The functions

$$
\frac{1}{n} \sum_{k=1}^{n} G\left(z, \alpha_{n k}\right)
$$

are harmonic in $R$ and uniformly limited on any closed set $C^{\prime}$ in $R$, hence form a normal family of harmonic functions in $R$. Let $\log \Phi_{1}(z)$ denote an arbitrary limit function of the family (75), the uniform limit on any closed set in $R$ of a suitably chosen subsequence of the family. The functions (75) are continuous in the two-dimensional sense on $C_{2}$ and vanish there, so the subsequence converges uniformly on any closed set in $\bar{R}$ containing no point of $C_{1}$. The limit function $\log \Phi_{1}(z)$ is continuous in the two-dimensional sense on $C_{2}$ and vanishes there. Consider now the function

$$
\begin{aligned}
& \frac{1}{n} \log \left|A_{n} \frac{\left(z-\beta_{n 1}\right) \cdots\left(z-\beta_{n, n+1}\right)}{\left(z-\alpha_{n 1}\right) \cdots\left(z-\alpha_{n n}\right)}\right| \\
& \quad-\frac{1}{n} \log \sum_{k=1}^{n} G\left(z, \alpha_{n k}\right)-\frac{1}{n} \log G(z, \infty),
\end{aligned}
$$

\footnotetext{
* The term Green's function is sometimes used for the negative of the present function.
} 
which when suitably defined in the points $\alpha_{n k}$ is harmonic without exception in the region exterior to and bounded by the curves $C_{2}$. The sequence (76) or any subsequence converges uniformly on any closed set interior to $R$ whenever that is true of the corresponding sequence or subsequence (75). By essentially the reasoning previously used in the proof of Theorem 12 we now have $\Phi(z) / \Phi_{1}(z) \equiv \gamma_{2}$ throughout $R$. Then the function $\Phi_{1}(z)$ is uniquely determined; from every subsequence of the sequence (75) can be extracted a new subsequence converging uniformly on any closed set in $R$ to the function $\log \Phi_{1}(z)$, hence the sequence (75) itself converges uniformly on any closed set in $R$ to the function $\log \Phi_{1}(z)$; the extension of Theorem 12 to the situation of Theorem 21 rather than of Theorem 1 is established.

In the extension of Theorem 13 to a region $R$ bounded by more than two Jordan curves, we naturally replace (46) by

$$
\operatorname{og} A_{n}=\sum_{k=1}^{n} G\left(\alpha_{n k}, \infty\right) ;
$$

since only the modulus of $A_{n}$ is of significance in (1), equation (77) is essentially equivalent to (46) under the hypothesis of Theorem 13. Of course the validity of (1) with the substitution (77) is to be established by the method indicated for the proof of (1) with the substitution (46) (op. cit., p. 274). The indicated extension of the remainder of $\$ \S 1-10$ now presents no difficulty, as the reader may verify; but in $\S 8$ a somewhat unusual convention is desirable regarding normal families of analytic functions (compare op. cit., §7.3).

We turn next to the question of replacing by more general sets the Jordan curves in Theorem 21 and in the corresponding results of $\$ \$ 1-10$. Theorem 5 extends without difficulty and with only minor modifications to the case that $R$ is bounded by a finite number of continua, none of which is a single point. There is no inherent difficulty in extending almost all of the discussion of the present paper to this more general case. However, with this more general hypothesis, such integrals as appear in (36) no longer have the meaning previously assigned, and points $\alpha_{n k}$ and $\beta_{n k}$ cannot be taken or proved uniformly distributed on $C_{1}$ and $C_{2}$ by the previous methods. There are then two possible procedures for determining the points $\alpha_{n k}$ and $\beta_{n k}$ : (i) consider integrals not over $C_{1}$ and $C_{2}$ but over sets of curves $\Gamma_{1}$ and $\Gamma_{2}$ in $R$ and approaching $C_{1}$ and $C_{2}$ respectively, choosing points $\alpha_{n k}$ and $\beta_{. l k}$ on the sets $\Gamma_{1}$ and $\Gamma_{2}$ (as in op. cit., §4.4); but of course these points $\alpha_{n k}$ and $\beta_{n k}$ then lie interior to $R$, and (1) may not be valid uniformly on $C_{1}$; (ii) if each point of $C_{1}$ and $C_{2}$ is a boundary point of the complement of $\bar{R}$, the harmonic function $U(x, y)$ in (36) may be uniformly approximated in $\bar{R}$ by functions harmonic throughout $\bar{R}$, by Lebesgue's theory of variable harmonic functions; these approximating 
functions may be handled by the original method of uniform distribution of points, and yield points $\alpha_{n k}$ and $\beta_{n k}$ exterior to $\bar{R}$ (compare op. cit., §4.4); this method can be used also for the appropriate points $\alpha_{n k}$ or $\beta_{n k}$ if only one of the sets $C_{1}$ and $C_{2}$ has the property that each of its points is a boundary point of the complement of $\bar{R}$. It will be noticed that method (i) enables us to use for $C$, the point set on which the given function is approximated, any closed bounded set not separating the plane having only a finite number of components of which none is a single point. By an extension of Theorem 5 we may here take $C$ an arbitrary closed bounded set whose complement is connected and possesses a Green's function.

Neither (i) nor (ii) enables us to establish uniform distribution on $C_{1}$ or $C_{2}$ of given points, and thus the necessity of the uniform distribution in the Corollary to Theorem 16, in Theorem 17, and in $\S 9$, is still unproved. In this connection there suggests itself another method: (iii) consider the integrals in a still more general sense, say in the sense of Stieltjes with respect to a variable defined on the boundary elements of the region; in the generalization of (36) this variable would be the conjugate of $U$, locally single-valued and continuous in $\bar{R}$. It seems to the writer probable that this method would be completely satisfactory both in determining points $\alpha_{n k}$ and $\beta_{n k}$ from such an equation as (36), and in establishing the uniform distribution of given points as in the Corollary to Theorem 16; but this method has never been completely carried out.

We have hitherto considered merely a single region $R$, but it is possible (op. cit., $\$ \S 8.7$ and 8.8 ) to have such a relation as (1) valid simultaneously interior to two mutually disjoint regions of the kind considered in Theorem 1. Indeed if these regions $R$ are preassigned, if the corresponding closed regions are mutually disjoint, and if no region separates another from the point at infinity, suitable harmonic functions can be used as generating functions to define the sequences $\alpha_{n k}$ and $\beta_{n k}$ so that (1) is valid on any closed set in each region $R$. Then our entire discussion of $\$ \S 1-10$ carries over to the new situation, with a single exception. The function $\Phi(z)$ of (1) is harmonic in each $R$, and a locus $C_{\gamma}$ occurs in each $R$. But it may occur that $\gamma$ as defined from the function $f(z)$ is limited by the behavior of $f(z)$ in one region $R$, and not by the function $f(z)$ in a second region $R$; for instance $f(z)$ may be analytic throughout the latter. Theorem 5 then fails to apply to this second region $R$, and the conclusions (22)-(26) that we have drawn from Theorem 5 apply to the first region $R$ but have not been established for the second region $R$. $^{*}$

* But for a series of interpolation (78) the principal results are valid also for the second region $R$. For various other specific examples the corresponding statement is true; the general question is still open. 
An important special case of Theorem 1 is that in which the $\alpha_{n k}$ and $\beta_{n k}$ are independent of $n$. Under these circumstances the interpolating functions $r_{n}(z)$ of Theorem 1 are the partial sums of a series of interpolation

$$
f(z)=a_{0}+a_{1} \frac{z-\beta_{1}}{z-\alpha_{1}}+a_{2} \frac{\left(z-\beta_{1}\right)\left(z-\beta_{2}\right)}{\left(z-\alpha_{1}\right)\left(z-\alpha_{2}\right)}+\cdots,
$$

and some of the results of Theorem 7 so far as it applies to the sequence $r_{n}(z)$ can be read off by elementary methods, without recourse to Theorem 5. For instance under the hypothesis of Theorem 1 we know (op. cit., §8.3) that the series in (78) converges to $f(z)$ uniformly on any closed set interior to $C_{\gamma}$, but by the definition of $\gamma$ can converge uniformly throughout the closed interior of no $C_{\gamma^{\prime}}, \gamma^{\prime}>\gamma$. There follows (op. cit., $\$ 3.4$, Theorem 5) the relation

$$
\limsup _{n \rightarrow \infty}\left|a_{n} / A_{n}\right|^{1 / n}=1 / \gamma .
$$

For the series (78) we deduce by inspection inequalities (2)-(4). Impossibility of the inequality sign in (2) and (4) follows at once from (1) and (79). Much of Theorem 7 for the series (78) is also a consequence of (1) and (79).

In generalizing the results of the present paper, it is also of interest to notice that points $\alpha_{n k}$ and $\beta_{n k}$ interior to $R$ may be admitted in restricted numbers, and that the equation (1) need not be supposed to hold uniformly on any closed set in $R$; under suitable circumstances it is sufficient if (1) holds uniformly on $C_{1}$ and on each $C_{\lambda}$ for $\lambda$ everywhere dense in the interval $\gamma_{2}<\lambda<\gamma_{1}$. A sufficiently general instance of this remark has already been elaborated elsewhere (op. cit., §8.5).

HARVARD UNIVERSITY,

Cambridge, Mass. 